\title{
An integral field spectroscopic survey for high redshift damped Lyman- $\alpha$ galaxies ${ }^{\star, \star \star}$
}

\author{
L. Christensen ${ }^{1}$, L. Wisotzki ${ }^{2}$, M. M. Roth ${ }^{2}$, S. F. Sánchez ${ }^{3}$, A. Kelz ${ }^{2}$, and K. Jahnke ${ }^{4}$ \\ ${ }^{1}$ European Southern Observatory, Casilla 19001, Santiago 19, Chile \\ e-mail: lichrist@eso.org \\ 2 Astrophysikalisches Institut Potsdam, An der Sternwarte 16, 14482 Potsdam, Germany \\ 3 Centro Astronómico Hispano Alemán de Calar Alto, Calle Jesús Durbán Remón 2,2 04004 Almería, Spain \\ 4 Max-Planck-Institut für Astronomie, Königstuhl 17, 69117 Heidelberg, Germany
}

Received 16 September 2006 / Accepted 8 March 2007

\section{ABSTRACT}

\begin{abstract}
Aims. We search for galaxy counterparts to damped Lyman- $\alpha$ absorbers (DLAs) at $z>2$ towards nine quasars, which have 14 DLAs and 8 sub-DLAs in their spectra.

Methods. We use integral field spectroscopy to search for Ly $\alpha$ emission line objects at the redshifts of the absorption systems.

Results. Besides recovering two previously confirmed objects, we find six statistically significant candidate Ly $\alpha$ emission line objects. The candidates are identified as having wavelengths close to the DLA line where the background quasar emission is absorbed. In comparison with the six currently known Ly $\alpha$ emitting DLA galaxies the candidates have similar line fluxes and line widths, while velocity offsets between the emission lines and systemic DLA redshifts are larger. The impact parameters are larger than $10 \mathrm{kpc}$, and lower column density systems are found at larger impact parameters.

Conclusions. Assuming that a single gas cloud extends from the QSO line of sight to the location of the candidate emission line, we find that the average candidate DLA galaxy is surrounded by neutral gas with an exponential scale length of $\sim 5 \mathrm{kpc}$.
\end{abstract}

Key words. galaxies: formation - galaxies: high-redshift - galaxies: quasars: absorption lines

\section{Introduction}

Galaxy counterparts to Damped Lyman- $\alpha$ systems (DLAs) seen in quasar (QSO) spectra have been suggested to be (proto)-disk galaxies with line of sight clouds of neutral gas with column densities $N(\mathrm{H} \mathrm{I})>2 \times 10^{20} \mathrm{~cm}^{-2}$ (Wolfe et al. 1986). Analyses of absorption line profiles have indicated that rotational components with velocities of $\sim 200 \mathrm{~km}^{-1}$ can be involved in these systems suggesting that DLAs reside in large disk galaxies (Prochaska \& Wolfe 1997; Ledoux et al. 1998a). On the other hand, numerical simulations show that in a hierarchical formation scenario merging proto-galactic clumps can also give rise to the observed line profiles (Haehnelt et al. 1998).

A large fraction of the neutral hydrogen present at $z>2$ is contained in high column density DLA systems (Lanzetta et al. 1995; Storrie-Lombardi \& Wolfe 2000; Péroux et al. 2001). In addition to the classical DLAs, clouds with column densities $10^{19}<N(\mathrm{H} \mathrm{I})<2 \times 10^{20} \mathrm{~cm}^{-2}$ also show some degree of damping wings, which is characteristic of DLA systems. It is suggested that sub-DLA systems contain a significant fraction of the neutral matter in the Universe (Péroux et al. 2003). Metallicity studies have shown that the properties of the sub-DLA systems are similar to those of DLA systems (Dessauges-Zavadsky et al. 2003), apart from the latter category having large ionisation corrections (Prochaska \& Herbert-Fort 2004).

* Based on observations collected at the Centro Astronómico Hispano Alemán (CAHA), operated by the Max-Planck Institut für Astronomie and the Instituto Astrofisica de Andalucia (CSIC).

$\star \star$ Full Fig. 2 is only available in electronic form at http://www. aanda.org
The association of DLAs with galaxies has been a subject of much study. Originally, either space-based or ground-based deep images were obtained to identify objects near the line of sight to the QSOs (Steidel et al. 1995; Le Brun et al. 1997; Warren et al. 2001). To confirm nearby objects as galaxies that are responsible for the DLA lines in the QSO spectra, follow-up spectra are needed to find the galaxy redshifts. At $z<1$, confirmations of 14 systems exist to date (Rao et al. 2003; Chen \& Lanzetta 2003; Lacy et al. 2003; Chen et al. 2005, and references therein), while at $z \gtrsim 2$ only 6 DLA galaxies are confirmed through spectroscopic observations of $\operatorname{Ly} \alpha$ emission from the DLA galaxies (Møller \& Warren 1993; Djorgovski et al. 1996; Møller et al. 1998; Leibundgut \& Robertson 1999; Møller et al. $2002,2004)$, three of which are located at the same redshifts as the QSOs themselves. Other techniques to identify DLA galaxies involve narrow-band imaging (e.g. Fynbo et al. 1999, 2000) or Fabry-Perot imaging. A Fabry-Perot imaging study of several QSO fields did not result in detections of emission from DLA galaxies (Lowenthal et al. 1995), while recently the same method was used to identify a few emission line candidates (Kulkarni et al. 2006).

Integral field spectroscopy (IFS) presents an alternative that provides images and spectra at each point on the sky simultaneously. This technique can be used to look for emission line objects at known wavelengths, but unknown spatial location. This technique is ideally suited to look for Ly $\alpha$ emission lines from the galaxies responsible for strong QSO absorption lines. At the Ly $\alpha$ wavelength corresponding to the redshift of the DLA system, the QSO emission has been absorbed, enabling us to locate emission line objects very near to the QSO line of sight. Because 
of the large column densities in DLAs and the resonant nature of Ly $\alpha$ photons the corresponding emission line may be offset in velocity space relative to the DLA line (e.g. Leibundgut \& Robertson 1999), but such an offset is not always observed (e.g. Møller et al. 2004).

IFS searches for emission from DLA galaxies towards two QSOs have resulted in upper limits for their fluxes (Petitjean et al. 1996; Ledoux et al. 1998b), while a sub-DLA galaxy previously known to be a $\operatorname{Ly} \alpha$ emitter was confirmed with IFS (Christensen et al. 2004). Here we present a survey using IFS to look for Ly $\alpha$ emitting DLA galaxies. Section 2 describes the sample of QSOs included in the survey, which are known previously to have DLAs and sub-DLAs in their spectra. Section 3 describes the observations and data reduction. In Sect. 4 the method of detecting emission line candidates is described. Section 5 presents the results and comments on each object. Properties of the Ly $\alpha$ emission candidates detected in the survey in relation to the six previously known Ly $\alpha$ emitting DLA galaxies are presented in Sect. 6. Section 7 summarises our findings. A flat cosmology with $H_{0}=70 \mathrm{~km} \mathrm{~s}^{-1} \mathrm{Mpc}^{-1}$, $\Omega_{\mathrm{m}}=0.3$, and $\Omega_{\Lambda}=0.7$ is used throughout.

This study, as well as previous ones that try to identify the host galaxies of DLA systems, can be biased since the galaxy observed at the right redshift likely belongs to the brightest emission line object close to the line of sight. In the case that the host galaxy is a much fainter galaxy in a group, it will not be identified correctly. In the remaining part of the paper, an "identified" DLA galaxy refers to observations that show (line) emission from independent observations, while the "candidates" are only reported in these IFS observations. Although extensive tests are done on the data to distinguish the candidates from potential artifacts, independent observations are needed to prove them as Ly $\alpha$ emitters connected with the DLAs.

\section{Sample selection}

We selected a number of DLA systems without previous detections of associated $\operatorname{Ly} \alpha$ emission. The selected QSOs with known DLAs were chosen based on the following criteria

1. $N(\mathrm{H} \mathrm{I})>2 \times 10^{20} \mathrm{~cm}^{-2}$

2. DLA redshift $(2<z<4)$

3. Northern hemisphere object.

The first criterion includes only classic DLA systems. Many QSO spectra show additional sub-DLA systems. Although their relationship to DLAs is still debated, these systems were included in the survey because of their probable physical association with galaxies.

To increase the sample size with a minimum number of pointings we preferentially selected QSOs with multiple DLAs. IFS covers a range of wavelengths, and correspondingly Ly $\alpha$ emission at a large range of redshifts in the line of sight for each QSO. However, in retrospect, this can affect the emission line detections, because extinction in foreground DLAs could obscure emission from background ones when the galaxies lie in the same line of sight. Hence, upper limits on detections of the higher redshift systems can be biased.

From the list of DLA systems compiled by S. Curran ${ }^{1}$, we found 66 QSOs matching these criteria in 2003. More recently, detections of DLAs in the Sloan Digitized Sky Survey QSO spectra have greatly increased the number of known DLAs

\footnotetext{
${ }^{1}$ http://www.phys.unsw.edu.au/ sjc/dla/
}

(Prochaska \& Herbert-Fort 2004; Prochaska et al. 2005). A systematic survey of all 66 objects would require a large amount of time with present instruments, so we selected a few systems based on their observability during the allocated observing runs. We avoided DLAs with Ly $\alpha$ absorption lines close to sky emission lines.

The total sample consists of 9 QSOs with a total number of 14 DLA systems as listed in Table 1. These QSOs have an additional 8 sub-DLAs which are included in the survey. Because of the small number of DLAs involved in the survey, a proper statistical study is not the aim of this paper. Instead we focus on a few systems to exploit the benefits of IFS for this kind of investigation.

To study the applicability of IFS in identifying DLA galaxies we initially observed DLA galaxies where Ly $\alpha$ emission had been reported previously in the literature. Two of these systems could be observed during our runs; Q2233+131 and PHL 1222, originally identified by Steidel et al. (1995), Djorgovski et al. (1996) and Møller et al. (1998). Both objects are reported to have extended Ly $\alpha$ emission (Fynbo et al. 1999; Christensen et al. 2004). Table 1 includes these two previously known Ly $\alpha$ emitting DLA and sub-DLA galaxies, although the criteria listed above are not satisfied. The absorption system towards Q2233+131 has a column density that classifies it as a sub-DLA. Unless otherwise noted, these two objects are kept separate from the detection of candidate emission line objects in the remainder of the paper.

Most of the DLAs in the IFS study lie towards bright QSOs $(R<19)$. This ensured that the PSF variations as a function of wavelength could be determined, which was necessary for the subtraction of the QSO emission. Bright QSOs had larger residuals from the subtraction of the continuum emission, which potentially affected out ability to recover emission line objects that were offset in velocity space and located closer than $1^{\prime \prime}$ to the QSO line of sight. However, tests with artificial objects showed that this was a minor problem for the data set (see Sect. 5.3).

\section{Observations and data reduction}

Using the Potsdam Multi Aperture Spectrophotometer (PMAS) mounted on the $3.5 \mathrm{~m}$ telescope at Calar Alto we observed the objects listed in Table 2 during several runs from 2002-2004. The PMAS integral field unit (IFU) was used in the standard configuration where 256 fibres are coupled to a $16 \times 16$ element lens array. During the observations each fibre covered $00^{\prime} .5 \times 0,{ }^{\prime} 5$ on the sky giving a field of view of $8^{\prime \prime} \times 8^{\prime \prime}$. Each fibre resulted in a spatial element (spaxel) represented by a single spectrum. The 256 spectra were recorded on a $2 \mathrm{k} \times 4 \mathrm{k}$ CCD which was read out in a $2 \times 2$ binned mode. With a separation of 7 pixels between individual spectra, the fibre to fibre cross-talk was negligible (less than $0.4 \%$ for an extraction of all 7 pixels). Detailed overviews of the PMAS instrument and capabilities are given in Roth et al. (2000, 2005).

For individual exposures a maximum time of 1800 s was used because of the large number of pixels affected by cosmic ray hits. Furthermore, because of varying conditions such as the atmospheric transmission and seeing, the total exposure time for each object was adjusted, or sometimes an observation was repeated under better conditions. The photometric conditions during observations were monitored in real time with the PMAS acquisition and guiding camera (A\&G camera) which is equipped with a $1 \mathrm{k} \times 1 \mathrm{k}$ CCD. Seeing values listed in Table 2 refer to the seeing FWHM measured in the A\&G camera images. Determining actual spectrophotometric conditions requires 
Table 1. List of the observed DLA and sub-DLA systems with column densities and metallicities taken from the literature.

\begin{tabular}{llllllll}
\hline \hline Coordinate name & Alt. name & $z_{\text {em }}$ & $z_{\text {abs }}$ & $\log N(\mathrm{HI})$ & {$[\mathrm{Fe} / \mathrm{H}]$} & {$[\mathrm{Si} / \mathrm{H}]$} & Ref. \\
\hline Q0151+048A & PHL 1222 & 1.93 & 1.934 & $20.36 \pm 0.10$ & & & $(1)$ \\
Q0953+4749 & PC 0953+4749 & 4.457 & 3.404 & $21.15 \pm 0.15$ & $>-2.178$ & $>-2.09$ & $(2,3)$ \\
& & & 3.891 & $21.20 \pm 0.10$ & $>-1.712$ & $>-1.60$ & \\
Q1347+112 & & & 4.244 & $20.90 \pm 0.15$ & $-2.50 \pm 0.17$ & $-2.23 \pm 0.15$ & \\
& & 2.679 & 2.471 & 20.3 & & $(4,5,6)$ \\
Q1425+606 & SBS 1425+606 & \multirow{2}{*}{3.163} & 2.05 & $20.3^{\dagger}$ & & & $(7)$ \\
Q1451+1223 & B1451+123 & 3.246 & 2.469 & $20.30 \pm 0.04$ & $-1.33 \pm 0.04$ & $>-1.03$ & $(8,9,10,21)$ \\
& & & 3.171 & $19.70 \pm 0.10$ & $-2.54 \pm 0.12$ & $-1.95 \pm 0.16$ & $(11,24)$ \\
& & & 2.254 & $20.30 \pm 0.15$ & $-1.87 \pm 0.16$ & $-1.62 \pm 0.15$ & $(19)$ \\
Q1759+7539 & GB2 1759+756 & 3.05 & 2.625 & $20.76 \pm 0.10$ & $-1.21 \pm 0.17$ & $-0.82 \pm 0.10$ & $(13,18,20)$ \\
& & & 2.91 & 19.8 & $-1.65 \pm 0.01$ & $-1.26 \pm 0.01$ & $(18)$ \\
Q1802+5616 & PSS J1802+5616 & 4.158 & 3.391 & $20.30 \pm 0.10$ & $-1.54 \pm 0.11$ & $>-1.55$ & $(23)$ \\
& & & 3.554 & $20.50 \pm 0.10$ & $-1.93 \pm 0.12$ & $>-1.82$ & \\
& & & 3.762 & $20.55 \pm 0.15$ & $-1.82 \pm 0.26$ & $>-1.74$ & \\
Q2155+1358 & PSS J2155+1358 & 4.256 & 3.811 & $20.35 \pm 0.20$ & $-2.19 \pm 0.23$ & $-2.04 \pm 0.22$ & $(3)$ \\
& & & 3.142 & $20.55 \pm 0.15$ & $>-1.68$ & $-1.26 \pm 0.17$ & $(3)$ \\
& & & 3.565 & $19.37 \pm 0.10$ & $-2.21 \pm 0.21$ & $-1.85 \pm 0.12$ & $(14,19)$ \\
Q2233+131 & & & 4.212 & $19.61 \pm 0.15$ & $-2.18 \pm 0.25$ & $-1.27 \pm 0.16$ & $(14,19)$ \\
& & 3.295 & 3.153 & 20.0 & $>-1.4 \pm 0.1$ & $>-1.04$ & $(15,16,17)$ \\
\hline
\end{tabular}

$\dagger$ Denotes a system where the reported $N(\mathrm{HI})$ needs to be confirmed through high resolution spectroscopy. References for either DLA redshifts or metallicities: (1) Møller et al. (1998); (2) Schneider et al. (1991); (3) Prochaska et al. (2003d); (4) Smith et al. (1986); (5) Wolfe et al. (1986); (6) Turnshek et al. (1989); (7) Wolfe et al. (1995); (8) Chaffee et al. (1994); (9) Stepanian et al. (1996); (10) Prochaska et al. (2002a); (11) Bechtold (1994); (12) Lanzetta et al. (1991); (13) Prochaska et al. (2001); (14) Péroux et al. (2003); (15) Steidel et al. (1995); (16) Lu et al. (1997); (17) Prochaska et al. (2003a); (18) Outram et al. (1999); (19) Dessauges-Zavadsky et al. (2003); (20) Prochaska et al. (2002b); (21) Lu et al. (1996); (22) Prochaska et al. (2003c); (23) Prochaska et al. (2003b); (24) Petitjean et al. (2000).

Table 2. Log of the observations. The last two columns show the average seeing during the integrations and the photometric conditions derived from the A\&G camera images.

\begin{tabular}{lllllll}
\hline \hline QSO & Date & $\begin{array}{l}\text { Exp.time } \\
(\mathrm{s})\end{array}$ & Grating & $\begin{array}{l}\lambda \text { coverage } \\
(\AA)\end{array}$ & Seeing & Conditions \\
\hline Q0151+048A & $2003-08-27$ & $5 \times 1800$ & V600 & $3500-5080$ & $0.8-1.2$ & stable \\
Q0953+4749 & $2004-04-16$ & $4 \times 1800$ & V300 & $3630-6980$ & 0.9 & stable \\
& $2004-04-21$ & $5 \times 1800$ & V300 & $3630-6980$ & 1.0 & non-phot. \\
Q1347+112 & $2004-04-20$ & $7 \times 1800$ & V300 & $3630-6980$ & 0.6 & non-phot. \\
Q1425+606 & $2004-04-19$ & $6 \times 1800$ & V300 & $3630-6750$ & 1.0 & stable \\
Q1451+1223 & $2004-04-17$ & $7 \times 1800$ & V300 & $3630-6980$ & 0.8 & non-phot. \\
Q1759+7539 & $2004-04-21$ & $7 \times 1800$ & V300 & $3630-6980$ & $1.0-1.5$ & non-phot. \\
Q1802+5616 & $2003-06-18$ & $2 \times 1800$ & V600 & $5100-6650$ & 1.0 & non-phot. \\
& $2003-06-20$ & $3 \times 1800$ & V600 & $5100-6650$ & 1.0 & non-phot. \\
& $2003-06-21$ & $4 \times 1800$ & V600 & $5100-6650$ & 1.8 & non-phot. \\
& $2003-06-22$ & $6 \times 1800$ & V600 & $5100-6650$ & 0.9 & stable \\
Q2155+1358 & $2003-08-26$ & $7 \times 1800$ & V600 & $4015-5610$ & 0.7 & stable \\
& $2003-08-27$ & $4 \times 1800$ & V600 & $4015-5610$ & 0.8 & non-phot. \\
Q2233+131 & $2002-09-03$ & $4 \times 1800$ & V300 & $3930-7250$ & $1.0-1.3$ & \\
& $2003-08-24$ & $6 \times 1800$ & V600 & $4000-5600$ & 0.6 & stable \\
& $2003-08-25$ & $4 \times 1800$ & V600 & $4000-5600$ & 0.7 & non-phot. \\
\hline
\end{tabular}

$\dagger$ Results from these observations are published in Christensen et al. (2004).

monitoring of the extinction coefficients which cannot be determined from the A\&G camera images. In Table 2 "stable" means that the photometry of the guiding star was constant within $1 \%$ during the observations.

The data were obtained using 2 gratings; one with 300 lines $\mathrm{mm}^{-1}$ and one with 600 lines $\mathrm{mm}^{-1}$, set at a chosen tilt to cover a selected wavelength range. The FWHM of the sky lines were measured to be 6.4 and $3.2 \AA$, respectively. Observations of spectrophotometric standard stars were carried out at the beginning and end of each night at the grating position used for the observations.

Data reduction was done by first subtracting an average bias frame. Before extracting the 256 spectra most cosmic ray hits were removed by the routine described in Pych (2004). A high threshold was chosen such that not all cosmic rays were removed, because a low threshold would also remove bright sky emission lines from some spectra. Remaining cosmic rays were removed from the extracted spectra using the program L.A. Cosmic (van Dokkum 2001). 
The locations of the spectra on the CCD were found from exposures of a continuum lamp, taken either before or after the science exposures, using a tracing algorithm developed for the IDL based PMAS data reduction package P3D (Becker 2002). The spectral extraction was done in two ways; a "simple extraction" that added all flux from each spectrum on the CCD (i.e. an extraction width of 7 pixels), and another method that took into account the profile of the spectrum on the CCD. This second method assumed that the spectral profiles were represented by Gaussian functions (Gaussian extraction) where the widths were allowed to vary with wavelength. Widths were determined by fits to each of the 256 spectra as a function of the wavelength, and the extraction used these width in combination with the centre found from the tracing algorithm. The Gaussian profile is an approximation because the profiles are not strictly Gaussian. The second method increased the signal-to-noise ratio by $>10 \%$ for faint objects and therefore unless otherwise noted, the results from the "Gaussian extraction" data cubes will be reported (see also Sánchez 2006).

After extraction, the spectra were wavelength calibrated using exposures of emission line lamps taken just before or after the observations. The wavelength calibration was done using the $\mathrm{P} 3 \mathrm{D}$ reduction tool. Comparisons with sky emission lines indicated an accuracy of the wavelength calibration of about $10 \%$ of the spectral resolution.

The spectra show a wavelength dependent fibre to fibre transmission. To correct for this effect, we extracted sky spectra obtained from twilight sky observations in the same way as the science observations. A one dimensional average sky spectrum was calculated. Each of the 256 spectra were divided by this average spectrum, and the fraction was fit by a polynomial function to reduce noise. These polynomials were used to flat field the science spectra.

Before combining individual frames, the extracted spectra were arranged into data cubes. Each data cube was corrected for extinction using an average extinction curve for Calar Alto (Hopp \& Fernandez 2002). The data cube combination took into account a correction for the differential atmospheric refraction using a theoretical prediction (Filippenko 1982). Relative spatial shifts between individual data cubes were determined from a two-dimensional Gaussian fit to the QSO PSF at a wavelength close to the strong DLA absorption lines.

Subtraction of the sky background was an iterative process because the locations of the emission line objects of interest were not known beforehand. PMAS, in the configuration used, does not have specifically allocated sky fibres. Instead, we selected 10-20 fibres uncontaminated by the QSO emission and the average spectrum was subtracted from all 256 spectra. Different spaxel selections were examined visually to select an appropriate sky spectrum which had no emission line or noisy pixels in the spectral region of interest.

Flux calibration was done in the standard way using observations of spectrophotometric standard stars. A one-dimensional spectrum of the standard star was constructed by co-adding flux from all 256 spaxels. This was used to create a sensitivity function that could be applied to each of the 256 spectra in the science exposures. For non-photometric nights the flux calibrated spectra were compared with QSO spectra from the literature to estimate photometric errors. However, no correction factor was applied to our spectra, because an intrinsic variability of the QSOs would make such scaling uncertain. For some cases we note in Sect. 5.5 that there are differences which could be caused by either non-photometric conditions or intrinsic variability.
For reference we show spectra of the target QSOs in Fig. 1. Where present, metal absorption lines corresponding to the highest column density DLAs are indicated. For QSOs with multiple DLAs lines only the DLA lines and their redshifts are indicated since the wavelength coverage does not include lines redwards of the QSO Ly $\alpha$ line. A detailed analysis of metal absorption lines requires higher resolution spectroscopy as presented elsewhere (e.g. Prochaska et al. 2003c; Péroux et al. 2003; Dessauges-Zavadsky et al. 2003). DLA redshifts derived from the metal absorption lines were consistent with those reported in the literature within the accuracy of the wavelength calibration of the data cubes.

\section{Search for DLA optical counterparts}

The observations covered the wavelengths of Ly $\alpha$ for all but one of the strong absorption systems listed in Table 1. Only the highest redshift sub-DLA system towards Q2155+1358 was not covered, i.e. the total number of systems included in this analysis is 21 DLA and sub-DLA systems.

\subsection{Expected sizes}

For this project we are only interested in small wavelength regions corresponding to $\operatorname{Ly} \alpha$ at the DLA redshifts, and thus the search for candidate galaxies could be carried out using customised narrow-band filters. IFS, on the other hand, has the advantage that the widths of the narrow-band filters can be adjusted to match those of the emission lines. Typically customised narrow-band filters have a larger transmission FWHM than the spectral resolution of IFS data. Hence, IFS allows detection of emission line objects with a higher signal-to-noise ratio than that possible with narrow-band filters. A disadvantage is the relatively small field of view of current IFUs, but this is not a serious concern. One can estimate the expected sizes of DLA galaxies (see Wolfe et al. 1986). Using a Schechter luminosity function and a power-law relation between the disk luminosity and gas radius given by the Holmberg relation $R / R^{*}=\left(L / L^{*}\right)^{\beta}$, one can calculate the expected impact parameter. Combining $\beta=0.26$ found for DLA galaxies at $z<1$ (Chen et al. 2005) with the luminosity function in for $z=3$ galaxies (Poli et al. 2003) one finds $R^{*} \approx 30 \mathrm{kpc}$. If DLA galaxies are similar to or fainter than $L^{*}$ galaxies this implies that DLA galaxies at $z>2$ are expected to lie closer than $\sim 4^{\prime \prime}$ from the QSO line of sight. The small field of view of IFUs is therefore well suited to search for $\operatorname{Ly} \alpha$ emission from DLA galaxies.

The estimated galaxy sizes are highly dependent on the parameters of the DLA galaxy luminosity and slope $\beta$. Most probably, high redshift DLA galaxies are not regular disks like those in the local universe. Numerical models of DLAs predict that the galaxies are mostly smaller than $10 \mathrm{kpc}$, while observations that give limits on the star-formation rates associated with DLAs suggest that DLAs are located in neutral gas around Lyman break galaxies (Wolfe \& Chen 2006). As DLA galaxies at $z>2$ are generally found to be fainter than an $L^{*}$ galaxy (Colbert \& Malkan 2002), we choose to consider only objects with impact parameters smaller than $30 \mathrm{kpc}$ for a more detailed analysis.

The impact parameters that we measure in the data correspond to the radially projected distances so the real distances to the absorber can be larger. Two candidates are found at impact parameters larger than $30 \mathrm{kpc}$, and they are likely not associated directly with the absorbers themselves. 

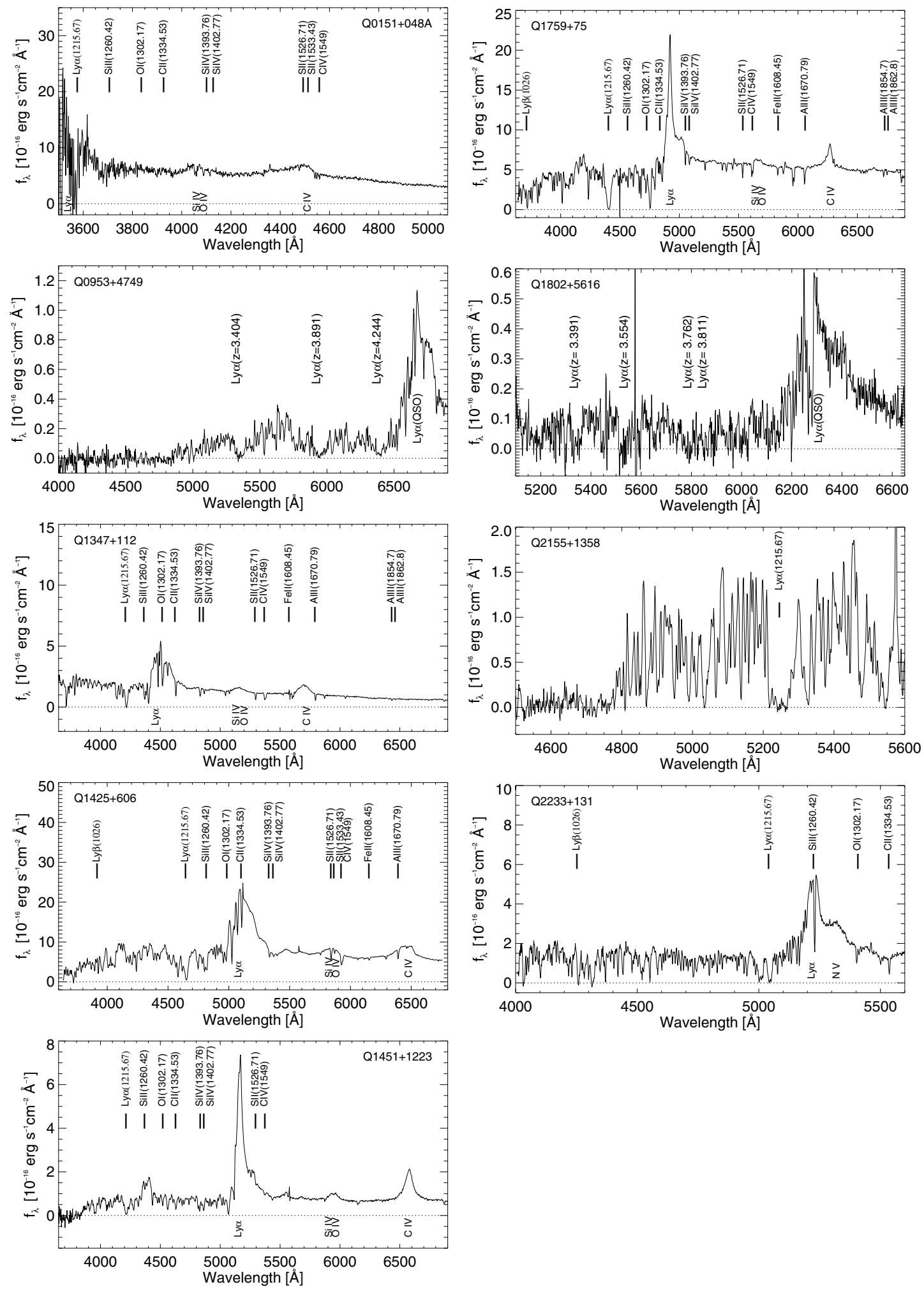

Fig. 1. QSO spectra extracted with a 3" radial aperture. No correction for Galactic reddening is applied. Metal absorption lines associated with the DLA systems are indicated. For those QSOs with multiple DLA lines only the DLA lines and their redshifts are indicated. Line identifications written below the spectra are QSO emission lines.

\subsection{Candidate selection}

Some Ly $\alpha$ emission lines from DLA galaxies are offset from the QSO- DLA line by $200 \mathrm{~km} \mathrm{~s}^{-1}$ (Møller et al. 2002), whereas Ly $\alpha$ emission from high redshift galaxies can have even larger offsets from the galaxy systemic redshift (Shapley et al. 2003).
We therefore chose to focus on regions in the data cubes with velocities ranging from approximately -1000 to $+1000 \mathrm{~km} \mathrm{~s}^{-1}$ from the DLA lines.

First, the reduced data cubes were stacked in a twodimensional frame and inspected visually around the DLA lines for emission line objects. When the spatial offset from the QSO 
is larger than the seeing, or alternatively when the QSO is very faint, emission line objects can be identified directly because of the ordering of the spectra in the stacked spectrum. Where no objects could be detected visually further sampling of the data cubes was necessary to increase the signal-to-noise ratio to detect candidate emission line objects. Inspections of the data cubes was done using the Euro3D visualisation tool (Sánchez 2004).

From the reduced, sky-subtracted and combined data cubes, narrow-band images were created with an initial width of 10-15 $\AA$ depending on the spectral resolution of the observations. A set of images was created offset by -10 to $+10 \AA$ from the DLA line to allow for possible velocity shifts of the Ly $\alpha$ emission line, and inspected visually for objects brighter than the background. If detected, spectra from these brighter regions were co-added and inspected for emission lines at the wavelength chosen in the narrow-band image. This step was necessary to discriminate between emission lines and individual noisy spectra. It is known that three blocks of 16 fibres, i.e. 48 fibres in an area of 1". 5 to the west in the field of view, have lower than average transmission. The effect of correcting for the total throughput was that these spectra had lower signal-to-noise ratios. When narrow-band images were created from the cubes, the higher variance in these spaxels could result in extreme values, seemingly inconsistent with the neighboring spaxels. Only by looking at the spectrum associated with a bright spaxel could it be determined if an emission line was present, or if the spectrum was just noisy. If an emission line was seen, a second pass narrow-band image was created using the value of the emission line width to increase the signal of the detection. A second pass one-dimensional spectrum was created after inspecting the narrow-band image for more bright spaxels surrounding the emission line candidate. This procedure was iterated until the signal in either narrow-band images or spectra did not increase. We found that an interactive visual identification of faint emission lines was more effective than an automatic routine.

To allow a better visual detection of emission line objects, the narrow-band images were interpolated to pixel scales $0 ! 2$ pixel $^{-1}$ as shown in Fig. 2. In all panels the images are $8^{\prime \prime}$ by $8^{\prime \prime}$, with orientation north up and east left. The left panels show interpolated images of the QSO at wavelengths near to the DLA line. Contours correspond to an image centered on the visually detected emission feature close to the DLA redshift. In the middle panels in Fig. 2 the plots are reversed, such that the image shows the emission line object and the contours correspond to the QSO narrow-band image. Here, the innermost contour corresponds to the seeing $F W H M$. To enhance the visibility of the candidates the QSO emission was subtracted from the data cubes before creating the images. This subtraction of the QSO emission was done using a simple approach (see Christensen et al. 2006). A scale factor was determined for each spaxel by dividing each spectrum by the extracted one-dimensional QSO spectrum. Using this scale factor, the QSO emission was subtracted, a process which retains objects with spectral characteristics different from the QSO in the data cube.

The spectra of the candidates are shown in the right hand column in Fig. 2. These are created by co-adding spectra from between 4 and 10 spaxels. The dotted line corresponds to the $1 \sigma$ noise level determined from a statistical analysis of the pixel values in the data cube, while the lower sub-panels show the background noise spectra in the data cubes, obtained from 4-10 background spaxels.
Properties of the candidate objects corresponding to those with spectra in Fig. 2 are listed in Table 3. Offsets in RA, Dec from the QSO and the corresponding projected distance at the DLA redshift are listed in Cols. 2-4. Emission lines were fit using ngaussfit in IRAF, redshifts listed in Col. 5 are derived. Fluxes in Col. 6 are derived from the Gaussian fits, and errors in the peak intensity, line width, and continuum placement are propagated to calculate the uncertainties. The fluxes have not been corrected for the Galactic extinction. The flux measurements and the associated errors indicate that most of the candidates are detected with a signal-to-noise ratio $<4 \sigma$. Column 7 gives the velocity difference between the systemic DLA redshift and the candidate Ly $\alpha$ emission lines. We integrate the signal-to-noise estimate over the emission line (Col. 8), $S / N_{\text {int }}=f /(\sqrt{N} \times \sigma)$, where $f$ is the line flux, $N$ is the number of pixels the emission line covers and $\sigma$ is the noise in adjacent wavelength intervals. Column 9 gives the observed emission line $F W H M$ after correcting for the instrumental resolution. Finally Col. 10 gives the significance classes of the candidate detection, which is explained in Sect. 5.1.

Columns 3 and 4 in Table 4 list the values of Galactic reddening towards each QSO (Schlegel et al. 1998), and the correction factors to be applied to the candidate fluxes for a Milky Way extinction curve (Fitzpatrick 1999).

\section{Results}

This section describes the classification of the emission line candidates. We estimate the contamination from spurious detections and from interlopers. Notes on each observed object are presented as well.

\subsection{Candidate significance class}

To estimate how reliable the candidate detection was, various tests were applied to the data cubes. The candidates were assigned a significance class: $1,2,3$, and 4 according to how many of the following tests were passed.

1. Instead of co-adding all data-cubes, two independent subsets of exposures were created, and the emission line candidate was visible in both sub-set combinations. In Sect. 5.5 these will be referred to as subcombinations.

2. The emission line candidate was visible in the "simple extractions", i.e. where a Gaussian profile was not assumed.

3. Emission line candidates were visible in the narrow-band images when the QSO spectrum was subtracted from the data cube.

4. Emission line objects that were directly visible in individual or combined data cubes, or in the stacked spectra.

In all cases, for a candidate to be considered further it was required to be detected above $3 \sigma$ in both narrow-band images and the associated spectra. The significance classes of the candidates are listed in Col. 10 in Table 3, and comments for each object are described in Sect. 5.5. Since the candidates were found from visual inspections of the data cubes, this classification was done to describe the candidates in a more qualitative manner. As the classes involve various tests on the data sets, this classification goes beyond the simple statistical significance in terms of to what $\sigma$ level the object is detected. 


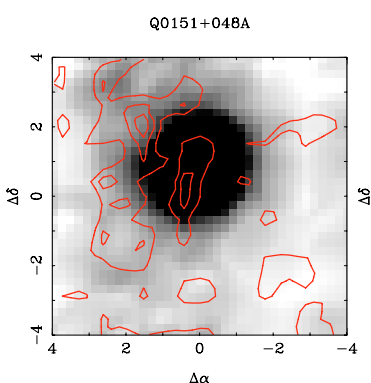

Q $0953+4749$

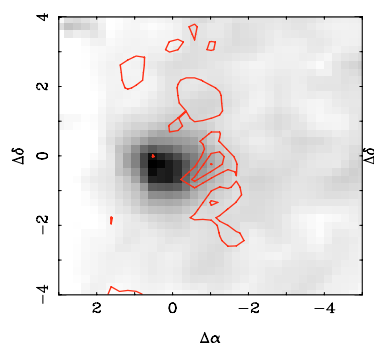

Q0953+4749

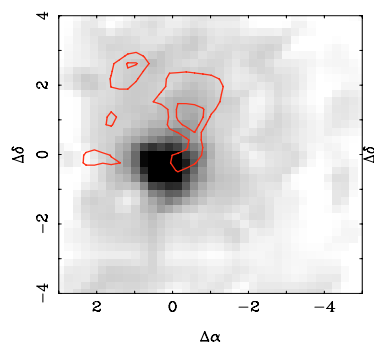

Q $0953+4749$

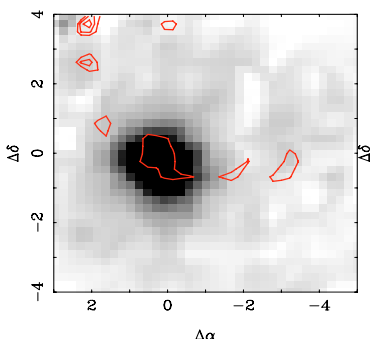

$1347+112$

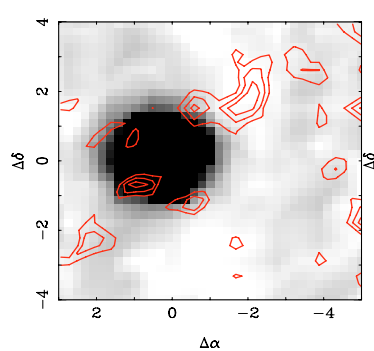

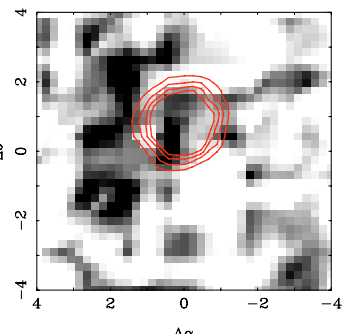

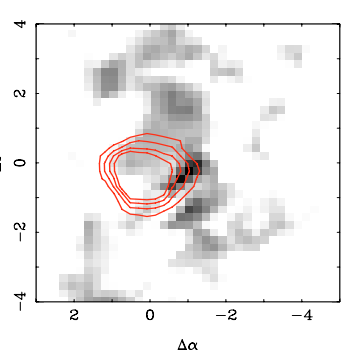

$z=3.903$

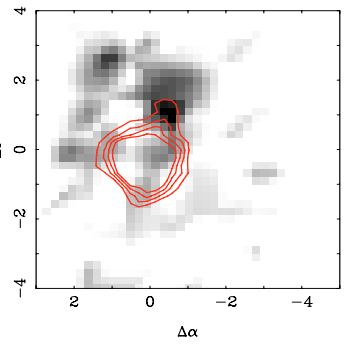

$z=4.244$

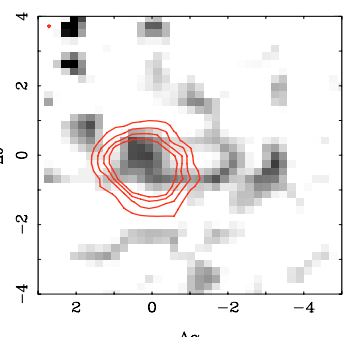

$\mathrm{z}=2.484$

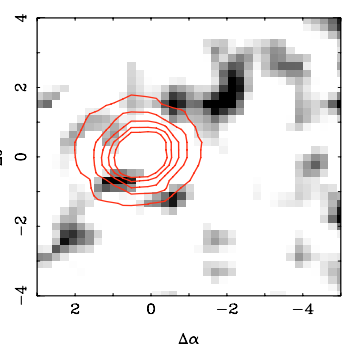

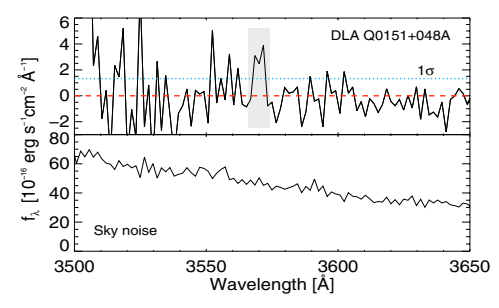
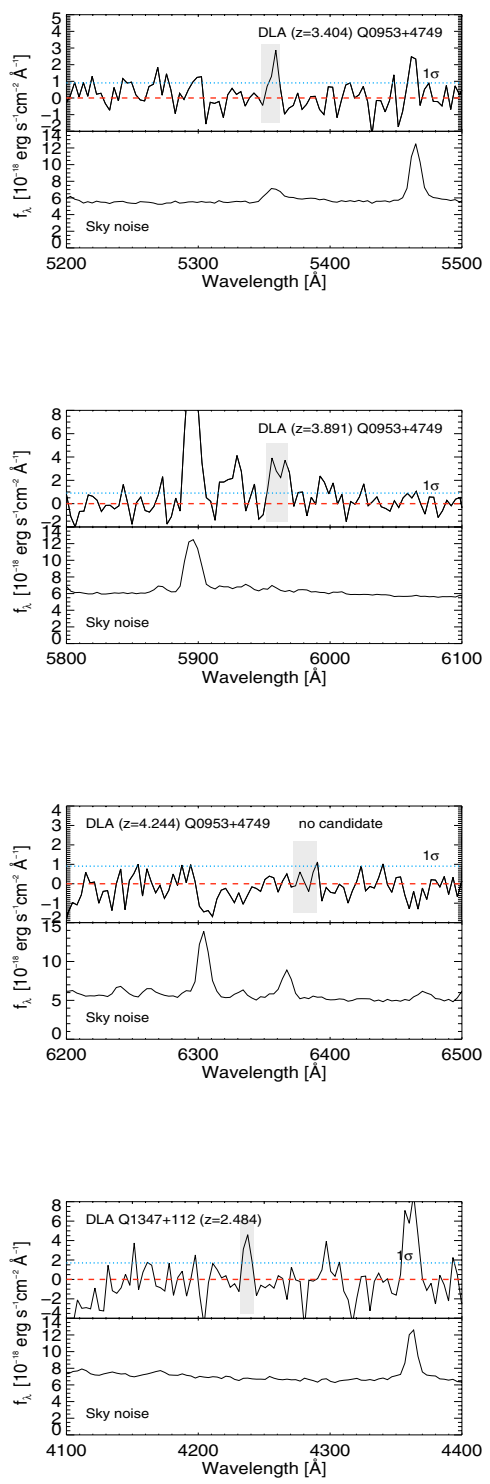

Fig. 2. Left panels: narrow-band images of the QSOs with overlayed contours of narrow-band images centered on the Ly $\alpha$ wavelengths of the DLAs. The images are 8 " square. Contour levels correspond to 2, 3, $4 \sigma$ levels above the background noise. Middle panels: the reverse, where the contours are arbitrary apart from the central one that shows the QSO seeing FWHM. Right hand panels: spectra of candidate emission line objects created from co-adding spectra from spaxels associated with the emission line candidates. The width of the grey bars over the emission lines correspond to the wavelength ranges of the narrow band images. The lines below the spectra show the background sky noise spectra determined from background spaxels. See the online version for plots of the remaining candidates.

\subsection{Non-detections}

In the data cubes where no candidates were found, we estimated the upper limits for the emission line fluxes. Spectra from spaxels within one seeing element (e.g. 4 spaxels corresponding to a seeing of $1^{\prime \prime}$ ) were co-added to create a one-dimensional spectrum. Artificial emission lines with varying line fluxes were added to this spectrum at the DLA wavelength, and Gaussian 
Table 3. Properties of candidate Ly $\alpha$ emission lines. Columns 2-4 list the offsets of the candidate in RA and Dec and in projected kpc at the Ly $\alpha$ emission redshifts (in Col. 5), respectively. Column 6 lists the integrated Ly $\alpha$ flux in units of $10^{-17} \mathrm{erg} \mathrm{cm}^{-2} \mathrm{~s}^{-1}$, and Col. 7 the velocity offset from the DLA redshift. Column 8 lists the integrated signal-to-noise ratio of the Ly $\alpha$ emission line, and Col. 9 gives the line width of the emission lines. Fluxes have not been corrected for Galactic extinction. Column 10 lists the significance class of the detections as described in Sect. 5.5. "Conf." implies candidates that were confirmed previously (Møller et al. 1998; Djorgovski et al. 1996).

\begin{tabular}{|c|c|c|c|c|c|c|c|c|c|}
\hline QSO & $\begin{array}{l}\Delta \mathrm{RA} \\
\left({ }^{\prime \prime}\right) \\
(2)\end{array}$ & $\begin{array}{l}\Delta \text { Dec } \\
\left({ }^{\prime \prime}\right) \\
(3)\end{array}$ & $\begin{array}{l}b \\
(\mathrm{kpc}) \\
(4)\end{array}$ & (5) & (6) & $\begin{array}{l}\Delta v \\
\left(\mathrm{~km} \mathrm{~s}^{-1}\right) \\
(7)\end{array}$ & $S / N_{\text {int }}$ & $\begin{array}{l}F W H M \\
\left(\mathrm{~km} \mathrm{~s}^{-1}\right) \\
(9)\end{array}$ & $\begin{array}{l}\text { significance } \\
\text { class } \\
(10)\end{array}$ \\
\hline Q0151+048A & $2.5: 0.4$ & $-2.5: 1.7$ & $3.3: 25.4$ & 1.9363 & $(150 \pm 70)$ & +225 & 7 & $280 \pm 220$ & conf. \\
\hline \multirow[t]{2}{*}{ Q0953+4749 } & -1.2 & -0.2 & 9.0 & 3.4041 & $(6.6 \pm 2.9)$ & 0 & 15 & $290 \pm 260$ & 2 \\
\hline & -0.5 & 1.8 & 11.1 & 3.9029 & $(4.9 \pm 2.1)$ & +730 & 16 & $570 \pm 230$ & 3 \\
\hline \multirow[t]{2}{*}{ Q1347+112 } & -2.0 & 1.5 & 20.2 & 2.4835 & $(3.5 \pm 1.9)$ & +1080 & 8 & $190 \pm 370$ & 3 \\
\hline & -4.1 & 0.4 & 34.3 & 2.0568 & $(4.2 \pm 3.0)$ & +670 & 7 & $610 \pm 210$ & 1 \\
\hline Q1425+606 & -4.1 & 0.4 & 32.3 & 2.8280 & $(8.5 \pm 3.1)$ & +80 & 14 & $590 \pm 220$ & 3 \\
\hline \multirow[t]{2}{*}{ Q1451+1223 } & -3.0 & 3.8 & 39.2 & 2.4764 & $(5.8 \pm 2.6)$ & +640 & 10 & $320 \pm 260$ & 3 \\
\hline & -1.0 & 2.8 & 22.5 & 3.1739 & $(3.1 \pm 2.0)$ & +210 & 7 & $<100$ & 3 \\
\hline \multirow[t]{2}{*}{ Q1759+7539 } & -1.4 & 3.5 & 30.0 & 2.6377 & $(5.8 \pm 3.0)$ & +1050 & 10 & $290 \pm 220$ & 2 \\
\hline & 0.1 & -1.2 & 9.4 & 2.9090 & $(6.0 \pm 2.9)$ & -80 & 21 & $240 \pm 260$ & 1 \\
\hline \multirow[t]{2}{*}{ Q1802+5616 } & -0.2 & -2.0 & 14.9 & 3.3820 & $(3.5 \pm 0.9)$ & -610 & 10 & $180 \pm 90$ & 4 \\
\hline & 0.2 & 1.8 & 12.9 & 3.7652 & $(4.6 \pm 1.9)$ & +200 & 7 & $<100$ & 2 \\
\hline \multirow[t]{2}{*}{ Q2155+1358 } & 0.7 & 1.2 & 10.2 & 3.3174 & $(9.4 \pm 3.0)$ & +100 & 9 & $780 \pm 210$ & 3 \\
\hline & 1.7 & 1.9 & 19.4 & 3.1461 & $(4.1 \pm 2.4)$ & +290 & 7 & $260 \pm 220$ & 3 \\
\hline Q2233+131 & 0.6 & 2.3 & 18.0 & 3.1543 & $(9.6 \pm 2.5)$ & +90 & 9 & $230 \pm 110$ & conf. \\
\hline
\end{tabular}

Table 4. Columns 2 and 3 give values of the Galactic reddening and the corresponding correction factor to be applied to the emission line candidates.

\begin{tabular}{lll}
\hline \hline QSO & $E_{B-V}$ & $f_{\text {frac }}$ \\
\hline Q0151+048A & 0.044 & 1.216 \\
Q0953+4749 $(z=3.4041)$ & 0.011 & 1.032 \\
Q0953+4749 $(z=3.9028)$ & & 1.028 \\
Q1347+112 $(z=2.4835)$ & 0.035 & 1.145 \\
Q1347+112 $(z=2.0568)$ & & 1.163 \\
Q1425+606 $(z=2.827)$ & 0.012 & 1.043 \\
Q1451+1223 $(z=2.4764)$ & 0.031 & 1.128 \\
Q1451+1223 (z=3.1739) & & 1.102 \\
Q1759+7539 (z=2.6377) & 0.053 & 1.220 \\
Q1759+7539 (z=2.91) & & 1.199 \\
Q1802+5616 $(z=3.3820)$ & 0.052 & 1.164 \\
Q1802+5616 $(z=3.7652)$ & & 1.145 \\
Q2155+1358 $(z=3.3174)$ & 0.067 & 1.222 \\
Q2155+1358 $(z=3.1461)$ & 0.067 & 1.237 \\
Q2233+131 & 0.068 & 1.240 \\
\hline
\end{tabular}

profile fits to these lines were used to estimate the detection level. The results are listed in Table 5. The varying limits are due to the wavelength dependent noise in the data cubes and in particular the presence of residuals from nearby sky emission lines.

\subsection{Experiments with artificial objects}

To investigate how the efficiency of the visual inspection depended on object properties, several experiments with artificial data cubes were made. Similar to artificial experiments for oneand two-dimensional data sets, artificial emission line objects were added to the data cubes. These objects were described by the location in RA and Dec, central wavelength, peak emission intensity, and the widths in RA, Dec and wavelength. For simplicity we assumed that an emission line object seen as a point source in the data cube could be represented by a Gaussian profile in each direction, i.e. described by a Gaussian ellipsoid in the data cube.
Table 5. DLA and sub-DLA systems where no candidate emission lines are found and $3 \sigma$ upper limits for the line fluxes. Fluxes are in units of $10^{-17} \mathrm{erg} \mathrm{cm}^{-2} \mathrm{~s}^{-1}$.

\begin{tabular}{lll}
\hline \hline QSO & $z_{\text {abs }}$ & $f_{\text {lim }}(3 \sigma)$ \\
\hline Q0953+4749 & 4.244 & 2.5 \\
Q1451+1223 & 2.256 & 4.0 \\
Q1802+5616 & 3.554 & 4.0 \\
Q1802+5616 & 3.811 & 2.2 \\
Q2155+1358 & 3.565 & 3.5 \\
Q2233+131 & 2.551 & 4.8 \\
\hline
\end{tabular}

We first tested completely simulated data cubes with statistical noise levels corresponding to the typical noise level in the combined data cubes. An emission line object with a flux of $5 \times 10^{-17} \mathrm{erg} \mathrm{cm}^{-2} \mathrm{~s}^{-1}$, a width of $800 \mathrm{~km} \mathrm{~s}^{-1}$, and spatial $F W H M$ of $1^{\prime \prime}$ was placed at a previously known wavelength. In the stacked spectra no objects could be seen immediately. The emission line was only identified after inspecting the data cube in the visualisation tool, and it was extracted and analysed in the same way as the real data. Similar tests were made by adding an emission line to a real data cube, where the background noise included the systematic noise as well as the pure Poissonian noise. These tests produced similar results for the faint emission lines with Ly $\alpha$ flux $f \sim 5 \times 10^{-17} \mathrm{erg} \mathrm{cm}^{-2} \mathrm{~s}^{-1}$, i.e. 1) the emission line flux could be recovered within uncertainties, 2) even at very small impact parameters the object could be found 3) the reconstructed PSF of the emission line object was irregular as in any of the images in Fig. 2.

We also tested an automatic routine where the re-detection of the artificial objects was done with no visual intervention. A set of narrow-band images were created in wavelength ranges around the artificial line. For the detection of an emission line the location was constrained to be within $\pm 10 \AA$ of the input central wavelengths. These images were smoothed and a twodimensional Gaussian profile was fit to the images. When an object was detected above a certain threshold, spaxels around the centre within the seeing $F W H M$ were co-added. A series of tests showed that the recovered flux was consistent within $1 \sigma$ errors 
for fluxes down to $f=5 \times 10^{-17} \mathrm{erg} \mathrm{cm}^{-2} \mathrm{~s}^{-1}$. In a typical data cube this was also the detection limit where $50 \%$ of the objects were re-identified, while the fraction of re-identified emission lines at this flux level from a visual inspection was larger.

Tests on the frequency of false detections in data cubes where no objects were present showed that simultaneous detections of objects in narrow-band images and associated spectra with $S / N>$ 3 occurred at a rate of less than $5 \%$ in a series of experiments. Therefore false detections cannot explain the large number of candidate objects.

\subsection{Field Ly $\alpha$ emitters}

We estimate here whether the detected candidates are likely to be field Ly $\alpha$ emitters having no association with the DLAs. Observations of high redshift objects have partly focused on detecting Ly $\alpha$ emission from galaxies to determine the global comoving star-formation rates (e.g. $\mathrm{Hu}$ et al. 1998, 2004). The density of Ly $\alpha$ emitters at $z \sim 3$ is estimated to be $15000 \mathrm{deg}^{-2} \Delta z^{-1}$ with line fluxes brighter than a mean of $f=1.5 \times 10^{-17} \mathrm{erg} \mathrm{cm}^{-2} \mathrm{~s}^{-1}$ (Hu et al. 1998; Kudritzki et al. 2000). From the luminosity function at $z \approx 3$ (van Breukelen et al. 2005), the expected number of field $\operatorname{Ly} \alpha$ emitters at a flux limit of $5 \times 10^{-17} \mathrm{erg} \mathrm{cm}^{-2} \mathrm{~s}^{-1}$ is $1.7 \times 10^{-4} \operatorname{arcsec}^{-2} \Delta z^{-1}$. In our survey, the 9 data cubes sample a total redshift interval of $\Delta z=21.55$ around $z \approx 3$. Statistically, it is expected that there are 0.2 field Ly $\alpha$ emitters in the whole sample presented here. Because these very faint lines are difficult to locate when the approximate wavelength is not known in advance, we did not look for field emission objects. The negligible number of expected field emitters furthermore shows that the emission candidates, if proved to be real, are unlikely to be interloping field Ly $\alpha$ emitters. They are much more likely to be associated with the DLA galaxies.

\subsection{Notes on individual objects}

This section explains the significance of the candidates for each individual QSO.

$Q 0151+045 A$. - This is a $z_{\mathrm{em}} \approx z_{\mathrm{abs}}$ system at $z \approx 1.93$. After flux calibration, the QSO spectrum is 2 mag brighter than that presented in Møller et al. (1998). The low instrument sensitivity at $3560 \AA$ combined with a variable extinction coefficient at Calar Alto makes the calibration uncertain.

Extended Ly $\alpha$ emission was observed in a region of $3^{\prime \prime} \times$ 6 " around the QSO mostly to the east of the QSO (Fynbo et al. 1999). Long slit spectroscopy along the long axis revealed velocity structures of $400 \mathrm{~km} \mathrm{~s}^{-1}$ that could be interpreted as a rotation curve (Møller 1999). In the IFS data extended emission is detected to some degree in Fig. 2, but not with the same detail as in the higher spatial resolution and larger field of view data in Fynbo et al. (1999). This is the only case in the sample where extended emission is found, but the signal is not strong enough to determine the velocity structure over the extended region. The spectrum shown in Fig. 2 is the total spectrum co-added from the whole nebula.

Q0953+4749. - This $z_{\mathrm{em}}=4.457 \mathrm{QSO}$ has three DLAs at $z_{\mathrm{abs}}=3.407,3.891$, and 4.244 (Bunker et al. 2003). A candidate associated with the lowest redshift DLA is visible in the narrowband image in Fig. 2. Independent subcombinations, the simple extraction, and the corresponding spectra show a faint emission line. This emission line coincides with a sky emission line $1.6 \AA$ away and could be due to sky subtraction errors, so we only assign this candidate a significance class of 2. A Ly $\alpha$ emission line from the DLA galaxy has been reported (A. Bunker, private comm.) but its line flux is below our detection limit. For the second DLA system at $z=3.891$ the object is present in subcombinations, the simple-extracted images, and in the extracted spectra. This candidate is assigned significance class 3 . No candidate is found for the highest redshift DLA to the detection limit reported in Table 5.

The locations of the candidates are compared to WFPC2 images obtained from the HST archive, but no continuum counterpart could be identified.

Q1347+112. - This $z_{\mathrm{em}}=2.679$ QSO has a DLA at $z_{\mathrm{abs}}=$ 2.471 and another possible one at $z_{\mathrm{abs}}=2.05$, which needs confirmation from spectroscopy at higher spectral resolution. An emission candidate for the $z=2.471$ DLA is visible in the subcombinations and the extracted one-dimensional spectra. In the simple extraction, the spectrum has a low signal-to-noise ratio and the emission feature in the spectrum is faint. We assign a significance class of 3 to this candidate. For the $z=2.0568$ DLA system we detect a candidate emission line object, but note an increase in the background noise shortwards of $3750 \AA$. The object is not seen in one of the subcombinations, nor the extracted spectra, and therefore the candidate is assigned a significance class of 1 .

A snapshot WFPC F555W image (Bahcall et al. 1992) obtained from the HST archive has a $5 \sigma$ limiting magnitude of $24.4 \mathrm{mag} \operatorname{arcsec}^{-2}$, but no continuum counterpart can be seen at the location of the candidate.

Q1425+606. - This $z_{\mathrm{em}}=3.163$ QSO has a DLA at $z_{\mathrm{abs}}=$ 2.827. Because this QSO is very bright, strong residuals within $1^{\prime \prime}$ from the QSO centre are present in the narrow-band image where the QSO emission is subtracted. A faint object offset by $\sim 4^{\prime \prime}$ to the west is visible in the narrow-band image in Fig. 2 . The candidate is present in subcombinations and in the constructed spectra and is assigned a significance class of 3. In PMAS data cubes, spaxels in the west region are more noisy than the average due to an overall lower transmission. Note that the tests suggest a good candidate, but the impact parameter $(>30 \mathrm{kpc})$ is large.

$Q 1451+1223$. - This $z_{\mathrm{em}}=3.246$ QSO has two DLAs at $z_{\mathrm{abs}}=2.469$ and $z_{\mathrm{abs}}=2.254$ and a sub-DLA at $z_{\mathrm{abs}}=3.171$. For the DLA system at $z=2.469$ an object appears after the QSO subtraction close to the centre. It is caused by residuals, since the spectrum has no emission lines at the expected wavelength. For the same DLA, a region $\sim 4^{\prime \prime}$ to the north west appears in both narrow-band imaging, subcombinations, simple extractions, and the constructed spectra. We therefore assign a significance class of 3 to it, but note that is has a large impact parameter $(39 \mathrm{kpc})$. For the $z=3.171 \mathrm{sub}-\mathrm{DLA}$ an object is detected to the north. Narrow-band images from subcombinations, and simple extractions show the emission line candidate, but the corresponding spectra have emission lines with very low signals. The candidate is assigned a significance class of 3 . No candidate is found for the $z=2.254$ DLA.

A deep optical broad-band image of the field surrounding this QSO was obtained by Steidel et al. (1995), who found no obvious candidates to the absorbers. Warren et al. (2001) found one candidate offset by 3 .'9 to the south-west of the QSO in a NICMOS image, but this object is outside the field of view of the IFS data. An HST/STIS archive image shows that the emission line candidates lie in regions where no continuum emitting counterpart is found.

Q1759+7539. - This $z_{\mathrm{em}}=3.05$ QSO has a DLA at $z_{\mathrm{abs}}=$ 2.625 and a sub-DLA at $z_{\mathrm{abs}}=2.91$. The candidate detected for the DLA system in Fig. 2 lies near the northern edge of the 
field of view and can be affected by flat field errors. Although it is bright, the candidate is not visible in both subcombinations, and is therefore assigned a significance class of 2 . A bright area 1." 8 south west of the QSO appears after the QSO emission is subtracted but it is likely due to residuals. It has no emission lines at the expected wavelength and is not considered further. The higher redshift sub-DLA system has an emission line candidate which is visible only after the QSO PSF has been subtracted from the final cube. However, the candidate is only visible in one out of two subcombinations and we assign this candidate a low significance class of 1 .

A NICMOS snapshot image showed no bright galaxies near the QSO to a limit corresponding to an $L^{*}$ galaxy (Colbert \& Malkan 2002).

Q1802+5616. - This $z_{\mathrm{em}}=4.158 \mathrm{QSO}$ has four DLAs at $z_{\mathrm{abs}}=3.391,3.554,3.762$, and 3.811. The candidate for the lowest redshift DLA system is directly visible in the reduced and combined data cube when looking at the stacked spectra. The candidate can also be identified in individual subcombinations and in the simple extracted spectrum. Therefore this candidate is assigned the class 4 . In a narrow-band image at the wavelength of $\operatorname{Ly} \alpha$ at $z=3.7652$ there is an emission region to the south (see Fig. 2) and the corresponding spectrum shows an emission feature. However, this line is coincident with a faint sky emission line, so this candidate is assigned the class 2 . No candidates are found for the other two DLA systems.

$Q 2155+1358$. - This $z_{\mathrm{em}}=4.256$ QSO has a DLA at $z_{\mathrm{abs}}=$ 3.316 and three sub-DLAs at $z_{\mathrm{abs}}=3.142,3.565$, and 4.212. The observations only cover $\operatorname{Ly} \alpha$ for the three lower redshift systems. IFS covering the highest redshift system has revealed a possible faint candidate emission line object (Francis \& McDonnell 2006). The candidate Ly $\alpha$ emission line associated with the DLA system is visible in independent subcombinations and in the simple extraction and is therefore assigned a high value of 3 . Because of the partial spatial overlap with the QSO, the emission from the QSO is subtracted to give a cleaned emission line object and the associated spectrum shown in Fig. 2. A candidate is found to the south for the $z=3.142$ sub-DLA system. This object is visible in the simple extraction, and subcombinations, but only one associated spectrum shows a clearly detected emission line. We assign a significance class of 3 to this candidate. No candidate is found for the $z=3.565$ sub-DLA.

$Q 2233+131$. - This $z_{\mathrm{em}}=3.295$ QSO has two sub-DLAs at $z_{\mathrm{abs}}=3.153$ and $z_{\mathrm{abs}}=2.551$. The galaxy responsible for the $z=3.153$ DLA was found by Steidel et al. (1995), and followup spectroscopy confirmed this by the detection of Ly $\alpha$ emission (Djorgovski et al. 1996). Previous IFS of this object suggested that the Ly $\alpha$ emission was extended (Christensen et al. 2004). This is not confirmed by the higher spectral resolution data included in this paper, although there appears to be some faint emission to the east of the object in Fig. 2. The new data and improved data reduction which optimises the signal-to-noise ratio, confirm the line flux Ly $\alpha$ line flux reported in Djorgovski et al. (1996). No candidate was found for the $z=2.551$ sub-DLA system, consistent with the upper limit from a deeper Fabry-Perot imaging analysis (Kulkarni et al. 2006).

\section{Properties of candidate DLA counterparts}

We proceed with a more detailed analysis of the properties of the detected candidate $\operatorname{Ly} \alpha$ emission lines. Only those candidates assigned values 3 and 4 are included. Of the eight good candidates, we reject two due to their large impact parameters

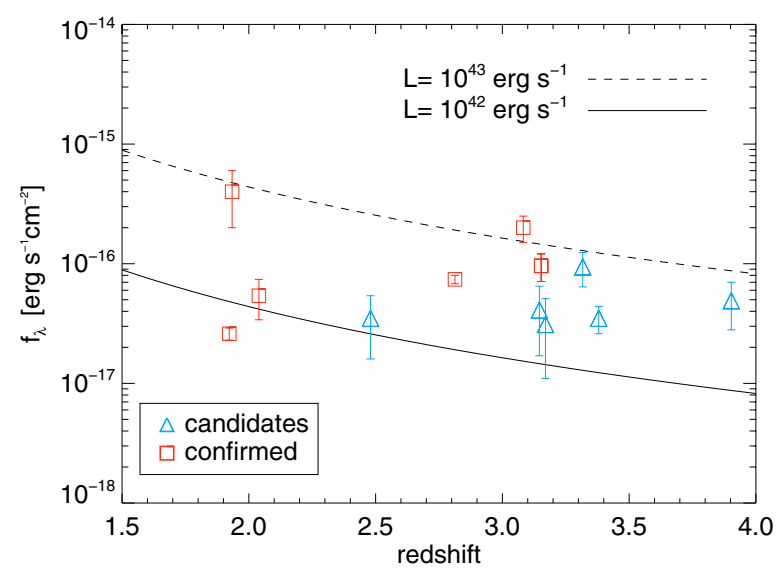

Fig. 3. Line fluxes of $\operatorname{Ly} \alpha$ emission objects as function of redshift, where square symbols represent already confirmed objects and triangles the candidates. The solid and dashed lines correspond to Ly $\alpha$ luminosities of $10^{42}$ and $10^{43} \mathrm{erg} \mathrm{s}^{-1}$, respectively.

(>30 kpc). However, since they fulfill the criteria for good candidates, they could instead belong to a brighter component in a group. The average redshift of all the DLAs in the whole sample is $\bar{z}_{\text {sample }}=3.13$ while that of the six remaining candidates is $\bar{z}_{\text {cand }}=3.23$, hence we find no preference for detection of either lower or higher redshift candidates. We emphasise that the candidates emission lines have fluxes that are detected at the $3 \sigma$ level, but with this in mind we compare their properties with those of confirmed Ly $\alpha$ emission lines from DLA galaxies.

\subsection{Line fluxes}

Figure 3 shows the inferred line fluxes of the candidates as a function of redshift. The triangles denote our candidates and square symbols indicate already confirmed objects from the literature (Møller \& Warren 1993; Djorgovski et al. 1996; Møller et al. 1998; Leibundgut \& Robertson 1999; Møller et al. 2002, 2004). This figure shows that the line fluxes for the candidates are similar to those for the previously confirmed ones, which have deeper observations and detection levels of 5-10 $\sigma$.

Fabry-Perot imaging studies of QSOs with DLAs have managed to reach similar or lower flux limits than our IFS survey (Lowenthal et al. 1995; Kulkarni et al. 2006). With their detection limit some objects should have been detected if the Ly $\alpha$ fluxes of DLA galaxies are around the level we find for the candidates and the confirmed objects. IFS is useful to look for emission lines as it allows us to adjust a posteriori the central wavelength, whereas in Fabry-Perot images, the emission line could fall at the wings of the filter where the transmission is lower. Another advantage of IFS observations is the knowledge of the spatial QSO PSF as a function of wavelength which allows a modeling and subtraction of the QSO emission (Wisotzki et al. 2003; Sánchez et al. 2004). This allows detection of emission lines even when they are superimposed on the QSO. Nevertheless we do not detect emission line candidates closer than about $1^{\prime \prime}$ from the QSO possibly due to subtraction residuals. The fact that the confirmed objects are found at smaller impact parameters compared to the candidates (Sect. 6.3) could indicate a bias. 


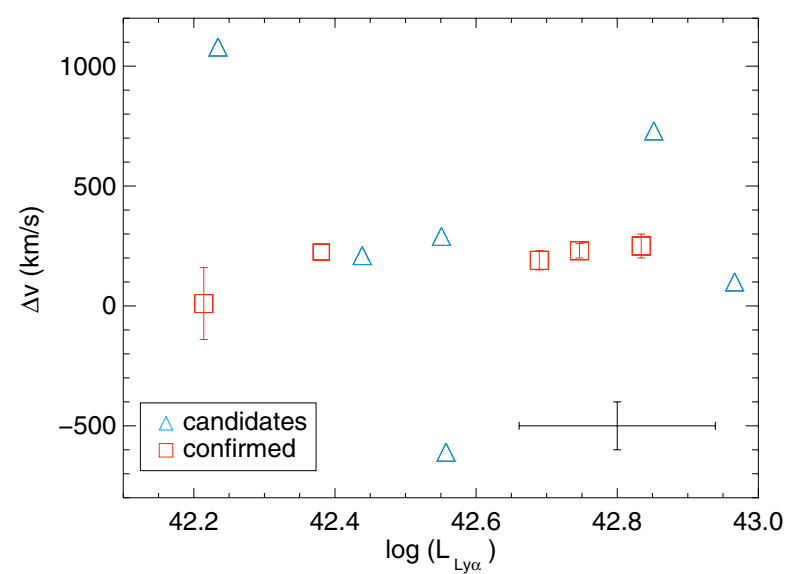

Fig. 4. Velocity differences between the Ly $\alpha$ emission lines and DLA redshifts as a function of the $\operatorname{Ly} \alpha$ luminosity. An average error bar for the $\operatorname{Ly} \alpha$ emission candidates is shown in the lower right corner.

\subsection{Velocity differences}

An anticorrelation is expected between the $\operatorname{Ly} \alpha$ luminosity and the velocity difference between the $\operatorname{Ly} \alpha$ emission line and optical emission lines (Weatherley et al. 2005). The resonant nature of Ly $\alpha$ causes a shift of the emission line towards slightly longer wavelengths where the photons can escape absorption. When a larger fraction of the blue part of the line profile is absorbed, the remaining emission line of lower luminosity will be more shifted in velocity compared to brighter ones. This explanation is supported by the study of Ly $\alpha$ emission lines from Lyman break galaxies (LBGs) (Shapley et al. 2003).

Figure 4 shows the velocity differences between Ly $\alpha$ emission lines and the DLA redshifts for the candidates as a function of the Ly $\alpha$ luminosity. There is no evidence for a correlation for the candidates. We note that the only candidate that shows a negative velocity offset is the best candidate in the sample; the $z=3.391$ DLA towards Q1802+5616. For the candidates we find an average velocity difference of $300 \pm 580 \mathrm{~km} \mathrm{~s}^{-1}$, which is similar to the velocity differences measured for LBGs; Pettini et al. (2001) find $560 \pm 410 \mathrm{~km} \mathrm{~s}^{-1}$ while a larger sample has $\Delta v=650 \mathrm{~km} \mathrm{~s}^{-1}$ between the Ly $\alpha$ emission line and lowionisation absorption lines (Shapley et al. 2003). In the case that DLAs are associated with bright galaxies we would expect to see large velocity offsets too. Furthermore, as the line of sight towards the emission line object and the QSOs differ by $10-30 \mathrm{kpc}$, a larger velocity offset can be expected due to differences in kinematics within the host and its environment. Instead, if the DLA galaxy resides in a group, the velocity offset will reflect the velocity dispersion in the group instead of being related to the host. In support of this idea, it has been shown that bright Lyman break galaxies at $z>2$ are surrounded by gas extending to large distances (Adelberger et al. 2005). Correlation studies have revealed that DLAs cluster on almost the same scale as LBGs (Cooke et al. 2006), indicating that a similar amount of gas is present in their environments.

Like flux-limited surveys, this IFS study selects the brightest emission component, and it is possible that the real absorbing galaxy is a fainter component in a group.

In the case that DLA galaxies are related to rotating large disks, it can be expected that the velocity difference increases with impact parameter but Fig. 5 shows no clear correlation. In three of the confirmed DLA galaxies optical emission lines have

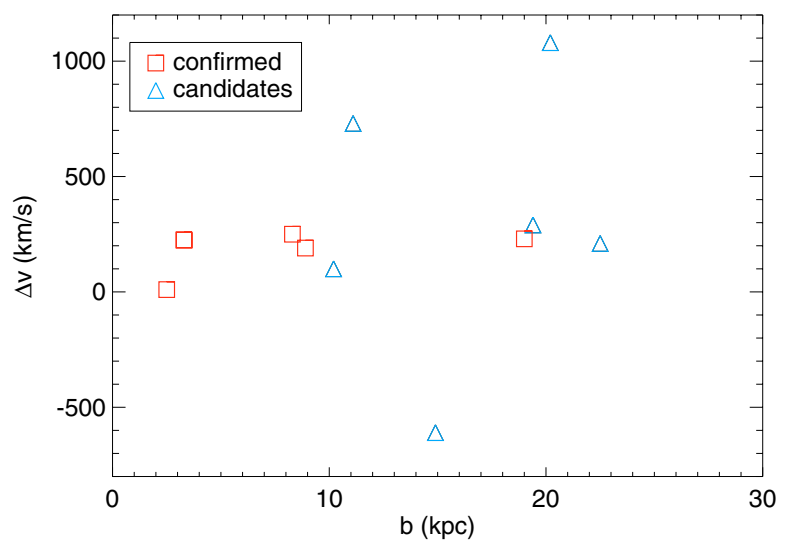

Fig. 5. Velocity differences between the $\operatorname{Ly} \alpha$ emission lines and DLA redshifts as a function of the impact parameter.

velocity differences between -200 and $30 \mathrm{~km} \mathrm{~s}^{-1}$ relative to Ly $\alpha$ (Weatherley et al. 2005). Some candidates have larger offsets, possibly affected more strongly by resonant scattering.

\subsection{HI extension}

The average impact parameter of $16 \mathrm{kpc}$ derived for the candidates is larger than that expected by numerical simulations which favor impact parameters of $b=3 \mathrm{kpc}$ for DLA galaxies, and have fewer than $25 \%$ with $b>10 \mathrm{kpc}$ at all redshifts (Haehnelt et al. 2000; Okoshi \& Nagashima 2005; Hou et al. 2005). Larger DLA galaxy sizes of $10-15 \mathrm{kpc}$ at $2<z<4$ are inferred by other simulations (Gardner et al. 2001). A possibility for the difference between observations and simulations is that the simulations assume a single disk scenario, while DLA galaxies could exist in groups (Hou et al. 2005). The real absorbing galaxy could be fainter and lie closer to the QSO line of sight than the detected candidate galaxy.

An anticorrelation between $N(\mathrm{HI})$ and the distance to the nearest galaxy is found in simulations (Gardner et al. 2001), but no analysis of this effect for observed DLA galaxies has been attempted. A trend for larger column density absorbers at smaller impact parameters was observed in a sample of DLA galaxies at $z<1$ (Rao et al. 2003). At lower column densities in the Ly $\alpha$ forest such an anticorrelation has been shown to exist (Chen et al. 1998, 2001). Observations of the galaxies giving rise to $\mathrm{Mg}$ II absorption lines showed an anticorrelation between the impact parameters and column densities of both Mg II and H I (Churchill et al. 2000), but recent observations of a larger sample have indicated that the correlation is not always present (Churchill et al. 2005).

We here investigate whether the candidates show a similar anticorrelation using the impact parameters for the candidates as a proxy for the sizes of neutral gas envelopes around proto galaxies. Assuming such a correlation is necessarily a rough approximation because large morphological differences between individual systems are expected (Rao et al. 2003; Chen \& Lanzetta 2003). Specifically, the possible presence of sub-clumps is neglected. 


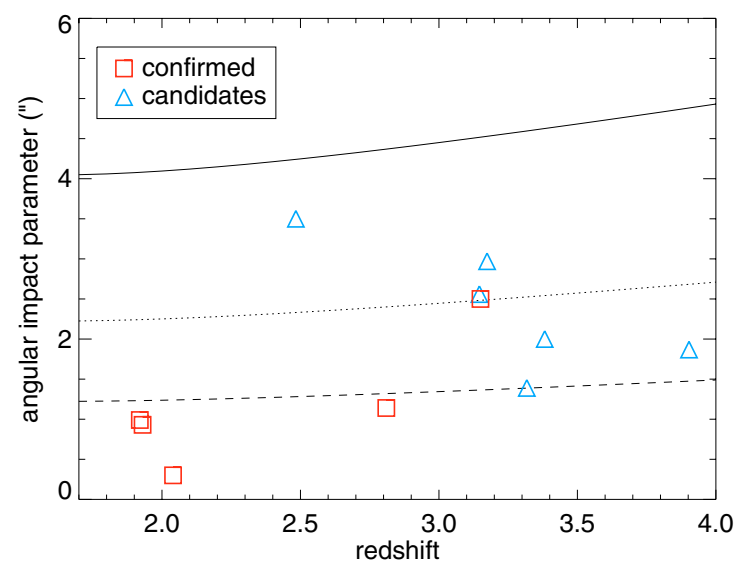

Fig. 6. Angular impact parameter vs. redshift. The solid line corresponds to the size-luminosity relation for an $L_{B}^{*}$ galaxy (Chen \& Lanzetta 2003), while the dotted and dashed lines correspond to galaxies with $B$ band luminosities $L_{B}=10 \% L_{B}^{*}$ and $L_{B}=1 \% L_{B}^{*}$, respectively. Squares are objects from the literature and triangles the candidates from this survey.

\subsubsection{DLA galaxy sizes and luminosities}

To analyse the sizes of DLA galaxies at $z<1$, Chen \& Lanzetta (2003) describe the extension of the neutral gas cloud associated with DLA galaxies as

$$
\frac{R}{R^{*}}=\left(\frac{L_{B}}{L_{B}^{*}}\right)^{t},
$$

assuming that DLA galaxies follow a Holmberg relation between galaxy sizes and luminosities. Their fit to the observed DLA galaxies gives $R^{*}=30 h^{-1} \mathrm{kpc}, t=0.26_{-0.06}^{+0.24}$, where $L_{B}^{*}$ corresponds to a galaxy with $M_{B}^{*}=-19.6$. Based on the morphologies and impact parameters Chen et al. (2005) argued that dwarf galaxies alone cannot represent the DLA galaxy population at $z<1$. In the case that the DLA galaxy population evolves from low luminosity objects at high redshifts to higher luminosity at lower redshifts, this will affect the expected impact parameters. In Fig. 6 the impact parameters of candidates and confirmed objects are shown as a function of their redshifts. Overlayed on this figure are curves for the size-luminosity relation derived for low redshift DLA galaxies. If DLA galaxies at high redshift follow the low redshift scaling relation, they comprise a mix of galaxy luminosities.

\subsubsection{Powerlaw profiles}

Using similar arguments as above one could expect that there is a relation between the impact parameter $b$, and the column density measured for the DLA. Figure 7 shows the $N(\mathrm{HI})$ measured for the DLA as a function of the impact parameters in kpc. We assume a similar scaling relation as in Eq. (1) for the impact parameter and $N(\mathrm{H}$ I), i.e.

$\frac{b}{b^{*}}=\left(\frac{N(\mathrm{HI})}{N(\mathrm{HI})^{*}}\right)^{\beta}$.

We set $\log N(\mathrm{H} \mathrm{I})^{*}=20.3$ and the error on the measured impact parameter is given by the fibre size of 0.5 , which corresponds to $\sim 4 \mathrm{kpc}$. A fit of the observed impact parameters for the candidates gives $b^{*}=15.9 \pm 1.4 \mathrm{kpc}$, and $\beta=-0.23 \pm 0.08$ as shown by the solid line, while for the confirmed objects, we find $b^{*}=12.0 \pm 3.7 \mathrm{kpc}$ and $\beta=-0.36 \pm 0.14$ as represented by the

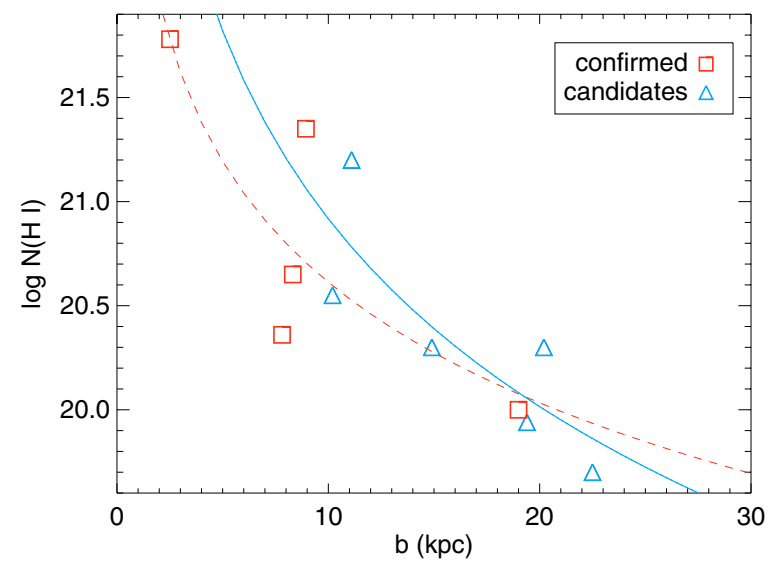

Fig. 7. Column density of neutral hydrogen as a function of impact parameters for the candidates and previously confirmed objects. The symbols are similar to the previous figures. The solid and dashed lines are fits to the power-law relation $b / b^{*}=\left(N / N^{*}\right)^{\beta}$ for the candidates and confirmed objects, respectively.

dashed line. The fits to the candidates and confirmed objects are consistent within $1 \sigma$ uncertainties.

\subsubsection{Exponential profiles}

Radio observations of the $21 \mathrm{~cm}$ emission from $\mathrm{H}$ I disks in the local Universe have shown that an exponential profile is a poor representation in the central part of disk galaxies, where the $21 \mathrm{~cm}$ flux density either stays constant or decreases towards the centre (e.g. Verheijen \& Sancisi 2001). However, at optical wavelengths disk galaxies are well represented by exponential profiles. Here we fit the impact parameter distribution by the relation

$N(\mathrm{HI})=N(\mathrm{HI})_{0} \exp (-b / h)$

where $h$ is the scale length, $N(\mathrm{HI})_{0}$ the central column density of a simple exponential disk, and $N(\mathrm{HI})$ is the column density measured for the DLAs. The resulting fit to all the candidates is shown by the solid line in Fig. 8, which has $\log N(\mathrm{HI})_{0}=$ $21.7 \pm 1.1 \mathrm{~cm}^{-2}$ and $h=5.1_{-1.3}^{+2.5} \mathrm{kpc}$. This result is similar within the uncertainties to a fit to the confirmed objects only $\left(\log N_{0}=\right.$ $21.7 \pm 1.1 \mathrm{~cm}^{-2}$ and $\left.h=4.5_{-1.4}^{+3.6} \mathrm{kpc}\right)$.

Local disk galaxies have optical scale lengths ranging from $\sim 2 \mathrm{kpc}$ to $\sim 6 \mathrm{kpc}$, and observations of the H I profile in low surface brightness galaxies have indicated scale lengths $>10 \mathrm{kpc}$ (Matthews et al. 2001). In contrast, higher redshift spiral galaxies in the HST Ultra Deep field have smaller optical scale lengths of 1.5-3 kpc (Elmegreen et al. 2005), possibly biased towards smaller values due to the easier detection of high surface brightness, high star formation rate regions. The question is how extended the gaseous envelopes are around these young galaxies. The impact parameters of the candidates suggest that high redshift DLAs reside far from the host galaxy, if not in a regular proto-galactic disk (Wolfe et al. 1986), then in a region of the same physical scale, possibly in merging clumps of gas surrounding the actual proto galaxy. In this picture, DLAs can be found far from the center of the parent galaxy.

The two objects that were originally discarded as candidates due to their large impact parameters $(>30 \mathrm{kpc})$ do not follow the relations for either the exponential or power-law profiles. Including them would make the scatter around the fit substantial. 


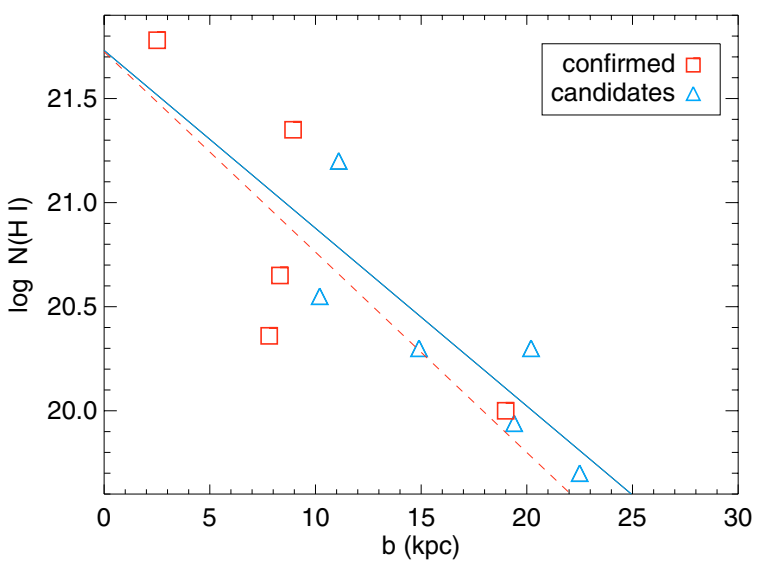

Fig. 8. Column density of neutral hydrogen as a function of impact parameter for the candidates and previously confirmed objects. The solid and dashed lines show the fit to the exponential relation $N(\mathrm{H} \mathrm{I})=N(\mathrm{HI})_{0} \exp (-b / h)$ for the candidates and confirmed objects, respectively.

\subsection{Metallicity effects on Lyo emission}

Using a space based imaging survey and follow up long-slit spectroscopic observations, Møller et al. (2004) found indications for a positive metallicity-Ly $\alpha$ luminosity relation, such that Ly $\alpha$ emission was preferentially observed in higher metallicity systems. They argued that this positive correlation could overpower the negative dust-Ly $\alpha$ luminosity effects which are expected to be strong in high metallicity environments (Charlot \& Fall 1993). Studies of Ly $\alpha$ emission from nearby star-forming galaxies have not revealed any correlations between metallicity and Ly $\alpha$ emission strength (Keel 2005). Differences in the velocity-metallicity relation between high and low redshift DLAs could be explained by higher redshift DLAs residing in lower mass galaxies (Ledoux et al. 2006), that have fainter Ly $\alpha$ emission.

In this context we investigate the distribution of metallicities for the emission line candidates in comparison to the total sample. We compare the cumulative distributions of metallicities $([\mathrm{Si} / \mathrm{H}])$ for the DLAs that have candidate Ly $\alpha$ detections with the metallicities of the remaining objects that have either no Ly $\alpha$ candidates or rejected candidates. The distributions in Fig. 9 show the fraction of DLAs with metallicities larger than a given value. Table 1 has several lower limits on $[\mathrm{Si} / \mathrm{H}]$ and Fig. 9 treats the limits as actual detections.

A two sided Kolmogorov Smirnov (KS) test gives a probability of $38 \%$ that the two samples have the same underlying distributions. A similar analysis for $[\mathrm{Fe} / \mathrm{H}]$ gives the same result. Hence, none of the tests give clear statistical evidence for a difference between the two populations. Only a small number of DLAs are included in this survey. For the two samples with $N_{1}$ and $N_{2}$ being the number of objects in each sample respectively we find $N_{1} N_{2} /\left(N_{1}+N_{2}\right)=4$. For the KS test to be statistically valid a value larger than 4 is required (Press et al. 1992), hence a few more objects are needed to make the test more statistically significant.

\subsection{Metallicity gradients}

In local galaxies the metallicities of $\mathrm{H}$ II regions are shown to decrease with increasing radial distance in the disk (Zaritsky et al. 1994). If DLAs arise in disks lower metallicities are expected at larger linear separations between the QSO line of sight and

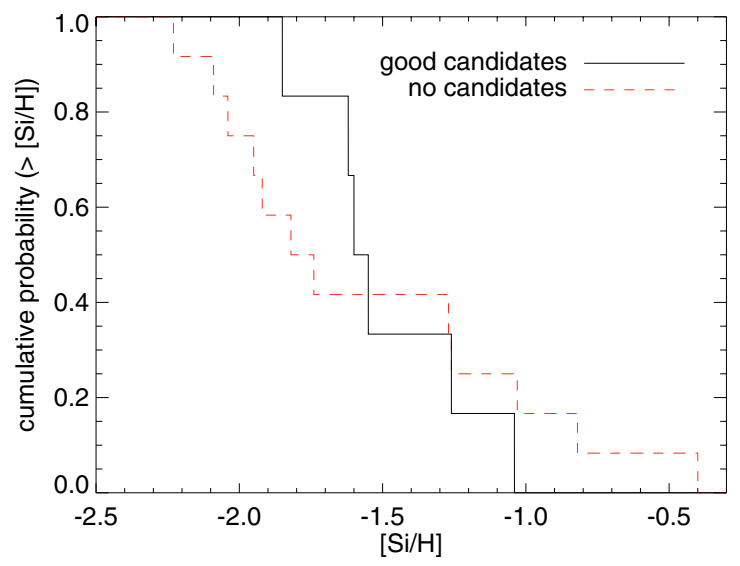

Fig. 9. Cumulative distribution of Si metallicities of the six good emission line candidates compared to the remaining part of the sample. The probability that the two distributions are similar is $38 \%$ estimated from a Kolmogorov-Smirnov test.

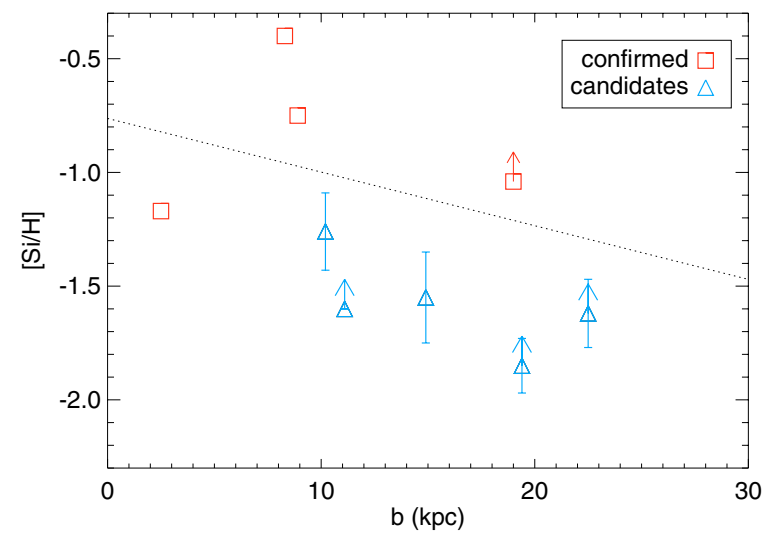

Fig. 10. DLA metallicities as a function of the impact parameters, where symbols shapes have similar meanings as in the previous figures. The dotted line shows a fit to all the objects excluding the limits. This line has a gradient of $-0.024 \pm 0.015 \mathrm{dex} \mathrm{kpc}^{-1}$. Data for the confirmed objects are either $[\mathrm{Si} / \mathrm{H}]$ of $[\mathrm{Zn} / \mathrm{H}]$ taken from Møller et al. (2004).

the galaxy centers. A comparison of absorption metallicities for 3 DLAs at $z<0.6$ with abundances derived from strong emission line diagnostics from the galaxy spectra revealed that gradients are likely present at at level $-0.041 \pm 0.012 \mathrm{dex} \mathrm{\textrm {kc } ^ { - 1 }}$ (Chen et al. 2005). Uncertainties in the gradients arise due to an unknown correction for dust depletion and also the inclination of the galaxy plays an important role due to projection effects. Metallicity differences due to differential depletion within a singe DLA galaxy can be strong as demonstrated by observations of a lensed QSO (Lopez et al. 2005).

Observations of nebular emission lines from seven galaxies at $2.0<z<2.5$ indicate an average solar metallicity (Shapley et al. 2004), with evidence for the presence of metallicity gradients (Förster Schreiber et al. 2006). If metallicity gradients exist for high redshift DLA galaxies, we would expect to see a tendency for higher metallicities for the DLA galaxies detected at smaller impact parameters. DLA galaxies are on the average fainter than LBGs detected in flux limited surveys (Møller et al. 2002). Combining this with a high redshift luminositymetallicity relation can imply low DLA metallicities without involving metallicity gradients.

Figure 10 shows the DLA metallicities as a function of the impact parameters. The line shows a fit to all objects ignoring 
those with lower limits on $[\mathrm{Si} / \mathrm{H}]$. This gradient is $-0.024 \pm$ 0.015 , i.e. is consistent with zero at the $2 \sigma$ level. The large scatter in the plot could be real and unrelated to gradients. Different DLAs exhibit a large range of star formation histories (Erni et al. 2006; Herbert-Fort et al. 2006), which makes it unreasonable to expect a smooth relation between metallicities and impact parameters for a sample of DLAs. Clearly more data are needed to determine the reality of any relation.

\section{Conclusions}

We have presented an integral field spectroscopic survey of 9 high redshift QSOs, which have a total of 14 DLA systems and 8 sub-DLA systems. We detect eight good candidates for Ly $\alpha$ emission lines from DLA and sub-DLA galaxies. Two of these are found at impact parameters larger than $30 \mathrm{kpc}$, and are not likely associated directly with the absorbing galaxy, but could be associated with galaxy groups in which the real absorbing galaxy resides. All candidates are detected at a statistically significant level in reconstructed narrow-band images as well as in the co-added one-dimensional spectra. Further observations will be useful to independently confirm the candidates at an even higher signal to noise ratio. We compare the properties inferred from the IFS data with those for previously spectroscopically confirmed Ly $\alpha$ emission lines from DLA galaxies reported in the literature. We find that line luminosities are similar to those of previously confirmed objects, that the average impact parameters are larger by a factor of $\sim 2$, and that some candidates have larger velocity offsets between the $\operatorname{Ly} \alpha$ emission line and the systemic redshift of the DLA system.

We analyse the distribution of DLA column densities as a function of impact parameters. Assuming that the average DLA galaxy is similar to a disk galaxy with an exponential profile, we show that it has a scale length of $5 \mathrm{kpc}$. Such a scale length is similar to disk scale lengths found for local spiral galaxies. This could imply that DLAs belong to large disks even at high redshifts as originally suggested by Wolfe et al. (1986). However, it is probably too simplistic to expect that high redshift DLAs reside in regular disks with similar structure to large local disks. DLA systems are generally not associated with luminous galaxies (e.g. Colbert \& Malkan 2002; Møller et al. 2002). The large impact parameters found for the candidates indicate that the distribution of H I clouds in DLA galaxies extends significantly beyond the optical sizes of fainter dwarf galaxies.

Furthermore, Wolfe \& Chen (2006) showed that high redshift DLAs do not reside in extended disks that follow the local Schmidt-Kennicutt law for star formation. The IFS results presented here suggest that the $\operatorname{Ly} \alpha$ emission is generally not extended, and that star formation takes place at a distance of several kpc from the DLA. Hence, we may speculate that DLAs arise in the outskirts of proto-galaxies, for example in clouds of neutral gas around LBGs. In this case one would expect a significant scatter in the relation between the impact parameter and column density of the DLA since neutral clouds could be distributed irregularly around the galaxy. Contrary to expectations, the objects show a small scatter around the relations in Figs. 7 and 8. Regardless of the distribution of neutral gas in DLA galaxies we conclude that there is a tendency to find a lower column density DLA with increasing impact parameter. Extending the investigation to include DLAs and sub-DLAs with $N(\mathrm{H} \mathrm{I})>$ $10^{19.6} \mathrm{~cm}^{-2}$ this tendency emerges for both the candidates and the confirmed objects.

The velocity offsets between the Ly $\alpha$ emission lines and the systemic redshifts of the DLAs are larger for half of the candidates compared to the confirmed objects. This could indicate an origin in groups of galaxies, where the DLA resides in a less luminous component than the galaxy detected in Ly $\alpha$. To determine whether resonant scattering affects the candidate Ly $\alpha$ lines more strongly and gives rise to larger velocity offsets than for the confirmed objects, observations of the corresponding optical emission lines which are shifted to the nearIR are required (e.g. Weatherley et al. 2005). Optical emission lines furthermore have the advantage of being less affected by dust absorption and therefore are better for estimating the starformation rates. Alternatively non-resonance UV lines such as C IV could be studied.

This survey was carried out with IFS on a 4-m class telescope, and the signals were generally near the detection limit. To verify this IFS method, it is necessary to get independent, higher signal-to-noise ratio spectra with a larger aperture telescope to confirm the existence of the emission lines.

Acknowledgements. This study was supported by the German Verbundforschung associated with the ULTROS project, grant No. 05AE2BAA/4. S.F. Sánchez acknowledges the support from the Euro3D Research Training Network, grant No. HPRN-CT2002-00305. K. Jahnke acknowledges support from DLR project No. 50 OR 0404. We thank the referee for useful suggestions that clarified the paper.

\section{References}

Adelberger, K. L., Shapley, A. E., Steidel, C. C., et al. 2005, ApJ, 629, 636 Bahcall, J. N., Maoz, D., Doxsey, R., et al. 1992, ApJ, 387, 56 Bechtold, J. 1994, ApJS, 91, 1

Becker, T. 2002, Ph.D. Thesis, Astrophysikalisches Institut Potsdam, Germany Bunker, A., Smith, J., Spinrad, H., Stern, D., \& Warren, S. 2003, Ap\&SS, 284, 357

Chaffee, F. H., Stepanian, J. A., Chavushian, V. A., Foltz, C. B., \& Green, R. F. 1994, BAAS, 26, 1338

Charlot, S., \& Fall, S. M. 1993, ApJ, 415, 580

Chen, H.-W., \& Lanzetta, K. M. 2003, ApJ, 597, 706

Chen, H.-W., Lanzetta, K. M., Webb, J. K., \& Barcons, X. 1998, ApJ, 498, 77

Chen, H.-W., Lanzetta, K. M., Webb, J. K., \& Barcons, X. 2001, ApJ, 559, 654

Chen, H.-W., Kennicutt, R. C., \& Rauch, M. 2005, ApJ, 620, 703

Christensen, L., Sánchez, S. F., Jahnke, K., et al. 2004, A\&A, 417, 487

Christensen, L., Jahnke, K., Wisotzki, L., \& Sánchez, S. F. 2006, A\&A, 459, 717

Churchill, C. W., Mellon, R. R., Charlton, J. C., et al. 2000, ApJ, 543, 577

Churchill, C. W., Kacprzak, G. G., \& Steidel, C. C. 2005, In Probing Galaxies through Quasar Absorption Lines

Colbert, J. W., \& Malkan, M. A. 2002, ApJ, 566, 51

Cooke, J., Wolfe, A. M., Gawiser, E., \& Prochaska, J. X. 2006, ApJ, 652, 994

Dessauges-Zavadsky, M., Péroux, C., Kim, T.-S., D’Odorico, S., \& McMahon, R. G. 2003, MNRAS, 345, 447

Djorgovski, S. G., Pahre, M. A., Bechtold, J., \& Elston, R. 1996, Nature, 382, 234

Elmegreen, B. G., Elmegreen, D. M., Vollbach, D. R., Foster, E. R., \& Ferguson, T. E. 2005, ApJ, 634, 101

Erni, P., Richter, P., Ledoux, C., \& Petitjean, P. 2006, A\&A, 451, 19

Filippenko, A. V. 1982, PASP, 94, 715

Fitzpatrick, E. L. 1999, PASP, 111, 63

Förster Schreiber, N. M., Genzel, R., Lehnert, M. D., et al. 2006, ApJ, 645, 1062 Francis, P. J., \& McDonnell, S. 2006, MNRAS, 656

Fynbo, J. U., Møller, P., \& Warren, S. J. 1999, MNRAS, 305, 849

Fynbo, J. U., Burud, I., \& Møller, P. 2000, A\&A, 358, 88

Gardner, J. P., Katz, N., Hernquist, L., \& Weinberg, D. H. 2001, ApJ, 559, 131

Haehnelt, M. G., Steinmetz, M., \& Rauch, M. 1998, ApJ, 495, 647

Haehnelt, M. G., Steinmetz, M., \& Rauch, M. 2000, ApJ, 534, 594

Herbert-Fort, S., Prochaska, J. X., Dessauges-Zavadsky, M., et al. 2006, PASP, 118,1077

Hopp, U., \& Fernandez, M. 2002, Calar Alto Newsletter No. 4,

http: //www. caha.es/newsletter/news02a/hopp/paper.pdf

Hou, J. L., Shu, C. G., Shen, S. Y., et al. 2005, ApJ, 624, 561

Hu, E. M., Cowie, L. L., \& McMahon, R. G. 1998, ApJ, 502, L99

Hu, E. M., Cowie, L. L., Capak, P., et al. 2004, AJ, 127, 563

Keel, W. C. 2005, AJ, 129, 1863

Kudritzki, R.-P., Méndez, R. H., Feldmeier, J. J., et al. 2000, ApJ, 536, 19

Kulkarni, V. P., Woodgate, B. E., York, D. G., et al. 2006, ApJ, 636, 30 
Lacy, M., Becker, R. H., Storrie-Lombardi, L. J., et al. 2003, AJ, 126, 2230 Lanzetta, K. M., McMahon, R. G., Wolfe, A. M., et al. 1991, ApJS, 77, 1 Lanzetta, K. M., Wolfe, A. M., \& Turnshek, D. A. 1995, ApJ, 440, 435

Le Brun, V., Bergeron, J., Boisse, P., \& Deharveng, J. M. 1997, A\&A, 321, 733

Ledoux, C., Petitjean, P., Bergeron, J., Wampler, E. J., \& Srianand, R. 1998a, A\&A, 337, 51

Ledoux, C., Theodore, B., Petitjean, P., et al. 1998b, A\&A, 339, L77

Ledoux, C., Petitjean, P., Fynbo, J. P. U., Møller, P., \& Srianand, R. 2006, A\&A, 457,71

Leibundgut, B., \& Robertson, J. G. 1999, MNRAS, 303, 711

Lopez, S., Reimers, D., Gregg, M. D., et al. 2005, ApJ, 626, 767

Lowenthal, J. D., Hogan, C. J., Green, R. F., et al. 1995, ApJ, 451, 484

Lu, L., Sargent, W. L. W., Barlow, T. A., Churchill, C. W., \& Vogt, S. S. 1996, ApJS, 107, 475

Lu, L., Sargent, W. L. W., \& Barlow, T. A. 1997, ApJ, 484, 131

Matthews, L. D., van Driel, W., \& Monnier-Ragaigne, D. 2001, A\&A, 365, 1

Møller, P. 1999, in Astrophysics with the NOT, ed. H. Karttunen, \& V. Piirola (University of Turku), 80

Møller, P., \& Warren, S. J. 1993, A\&A, 270, 43

Møller, P., Warren, S. J., \& Fynbo, J. U. 1998, A\&A, 330, 19

Møller, P., Warren, S. J., Fall, S. M., Fynbo, J. U., \& Jakobsen, P. 2002, ApJ, 574, 51

Møller, P., Fynbo, J. U., \& Fall, S. M. 2004, A\&A, 422, L33

Okoshi, K., \& Nagashima, M. 2005, ApJ, 623, 99

Outram, P. J., Chaffee, F. H., \& Carswell, R. F. 1999, MNRAS, 310, 289

Péroux, C., Storrie-Lombardi, L. J., McMahon, R. G., Irwin, M., \& Hook, I. M. 2001, AJ, 121, 1799

Péroux, C., Dessauges-Zavadsky, M., D’Odorico, S., Kim, T., \& McMahon, R. G. 2003, MNRAS, 345, 480

Petitjean, P., Pecontal, E., Valls-Gabaud, D., \& Charlot, S. 1996, Nature, 380, 411

Petitjean, P., Srianand, R., \& Ledoux, C. 2000, A\&A, 364, L26

Pettini, M., Shapley, A. E., Steidel, C. C., et al. 2001, ApJ, 554, 981

Poli, F., Giallongo, E., Fontana, A., et al. 2003, ApJ, 593, L1

Press, W. H., Teukolsky, S. A., Vetterling, W. T., \& Flannery, B. P. 1992, Numerical recipes in FORTRAN. The art of scientific computing (Cambridge: University Press), 2nd edn.

Prochaska, J. X., \& Herbert-Fort, S. 2004, PASP, 116, 622

Prochaska, J. X., \& Wolfe, A. M. 1997, ApJ, 487, 73

Prochaska, J. X., Wolfe, A. M., Tytler, D., et al. 2001, ApJS, 137, 21

Prochaska, J. X., Henry, R. B. C., O’Meara, J. M., et al. 2002a, PASP, 114, 933
Prochaska, J. X., Howk, J. C., O'Meara, J. M., et al. 2002b, ApJ, 571, 693 Prochaska, J. X., Castro, S., \& Djorgovski, S. G. 2003a, ApJS, 148, 317 Prochaska, J. X., Castro, S., \& Djorgovski, S. G. 2003b, ApJS, 148, 317 Prochaska, J. X., Gawiser, E., Wolfe, A. M., Castro, S., \& Djorgovski, S. G. 2003c, ApJ, 595, L9

Prochaska, J. X., Gawiser, E., Wolfe, A. M., Cooke, J., \& Gelino, D. 2003d, ApJS, 147, 227

Prochaska, J. X., Herbert-Fort, S., \& Wolfe, A. M. 2005, ApJ, 635, 123

Pych, W. 2004, PASP, 116, 148

Rao, S. M., Nestor, D. B., Turnshek, D. A., et al. 2003, ApJ, 595, 94

Roth, M. M., Bauer, S., Dionies, F., et al. 2000, in Proc. SPIE, 4008, 277

Roth, M. M., Kelz, A., Fechner, T., et al. 2005, PASP, 117, 620

Sánchez, S. F. 2004, AN, 325, 167

Sánchez, S. F. 2006, AN, 327, 850

Sánchez, S. F., Garcia-Lorenzo, B., Mediavilla, E., González-Serrano, J. I., \& Christensen, L. 2004, ApJ, 615, 156

Schlegel, D. J., Finkbeiner, D. P., \& Davis, M. 1998, ApJ, 500, 525

Schneider, D. P., Schmidt, M., \& Gunn, J. E. 1991, AJ, 101, 2004

Shapley, A. E., Steidel, C. C., Pettini, M., \& Adelberger, K. L. 2003, ApJ, 588, 65

Shapley, A. E., Erb, D. K., Pettini, M., Steidel, C. C., \& Adelberger, K. L. 2004, ApJ, 612, 108

Smith, H. E., Cohen, R. D., \& Bradley, S. E. 1986, ApJ, 310, 583

Steidel, C. C., Pettini, M., \& Hamilton, D. 1995, AJ, 110, 2519

Stepanian, J. A., Chavushian, V. H., Chaffee, F. H., Foltz, C. B., \& Green, R. F. 1996, A\&A, 309, 702

Storrie-Lombardi, L. J., \& Wolfe, A. M. 2000, ApJ, 543, 552

Turnshek, D. A., Wolfe, A. M., Lanzetta, K. M., et al. 1989, ApJ, 344, 567 van Breukelen, C., Jarvis, M. J., \& Venemans, B. P. 2005, MNRAS, 359, 895 van Dokkum, P. G. 2001, PASP, 113, 1420

Verheijen, M. A. W., \& Sancisi, R. 2001, A\&A, 370, 765

Warren, S. J., Møller, P., Fall, S. M., \& Jakobsen, P. 2001, MNRAS, 326, 759

Weatherley, S. J., Warren, S. J., Møller, P., et al. 2005, MNRAS, 358, 985

Wisotzki, L., Becker, T., Christensen, L., et al. 2003, A\&A, 408, 455

Wolfe, A. M., \& Chen, H.-W. 2006, ApJ, 652, 981

Wolfe, A. M., Turnshek, D. A., Smith, H. E., \& Cohen, R. D. 1986, ApJS, 61, 249

Wolfe, A. M., Lanzetta, K. M., Foltz, C. B., \& Chaffee, F. H. 1995, ApJ, 454, 698

Zaritsky, D., Kennicutt, R. C., \& Huchra, J. P. 1994, ApJ, 420, 87 
L. Christensen et al.: An IFS survey for high- $z$ DLA galaxies, Online Material $p 1$

\section{Online Material}


L. Christensen et al.: An IFS survey for high-z DLA galaxies, Online Material $p 2$
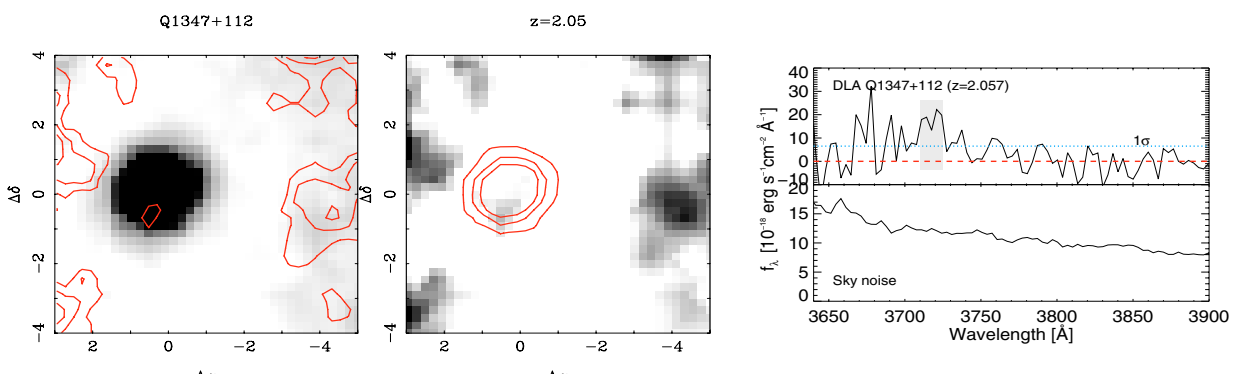

Q1425+606

$z=2.828$
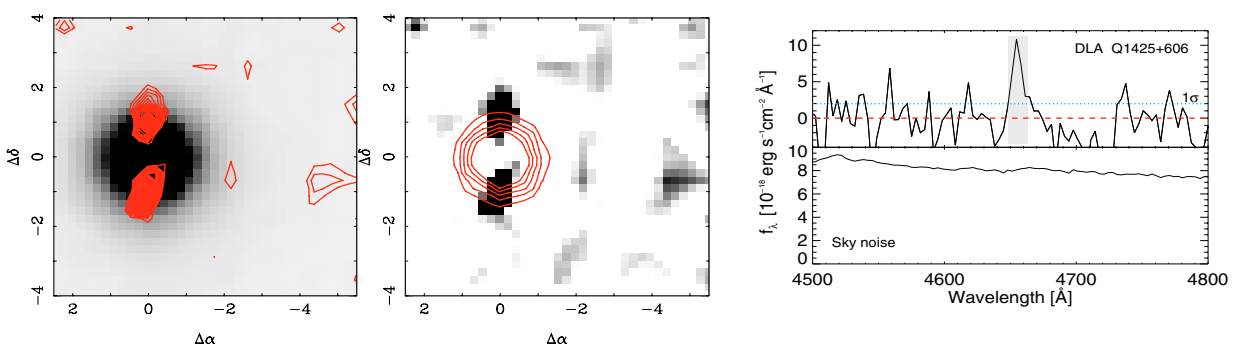

Q1451+1223

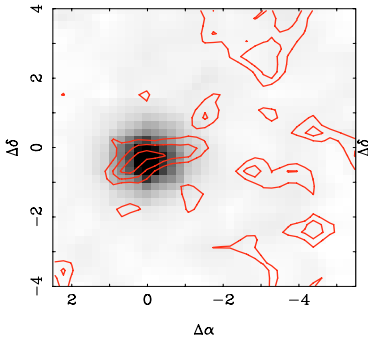

$z=2.476$
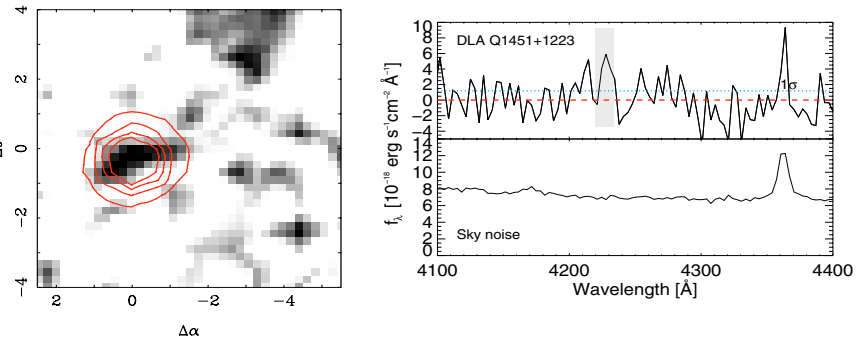

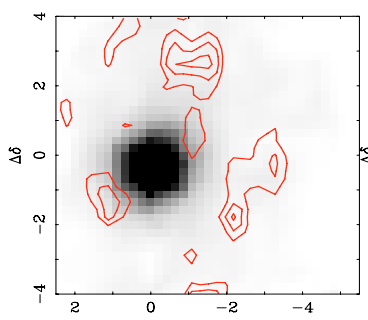

$\Delta \alpha$

Q1451+1223
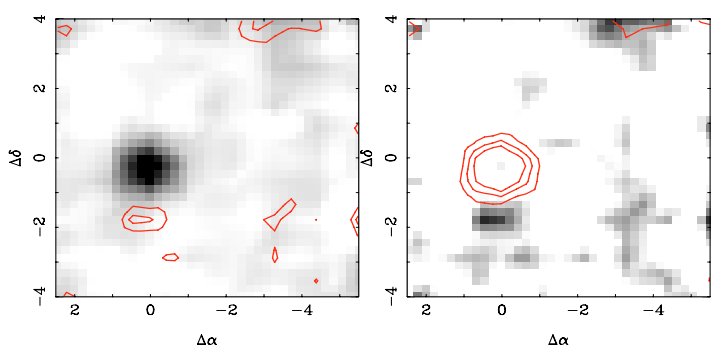

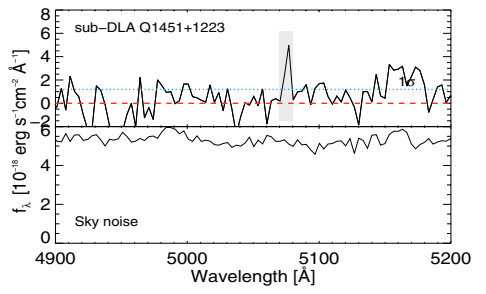

Fig. 2. continued. 
L. Christensen et al.: An IFS survey for high- $z$ DLA galaxies, Online Material $p 3$

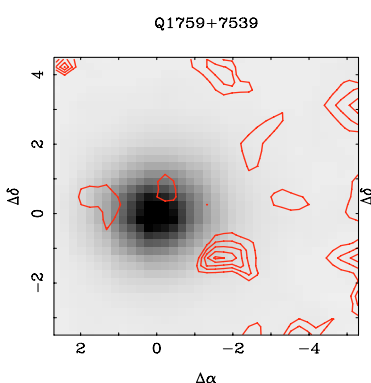

$1759+7539$

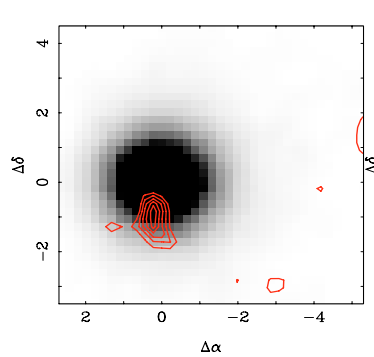

Q1802+5616

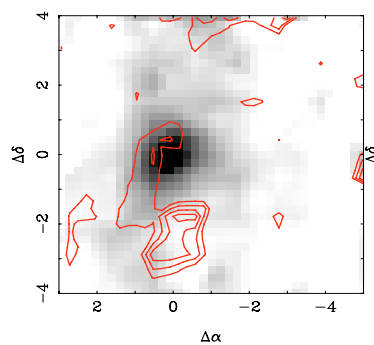

Q1802+5616

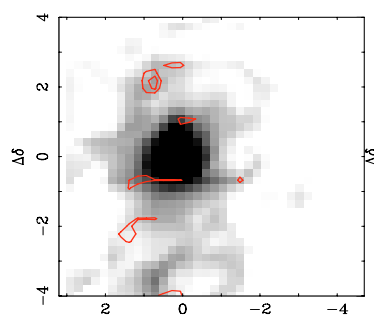

$\Delta \alpha$

Q1802+5616

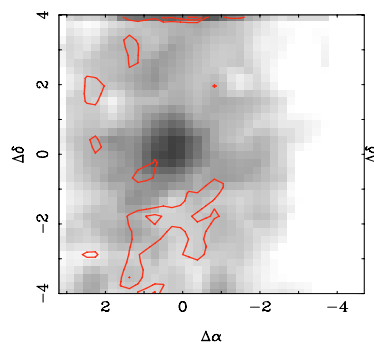

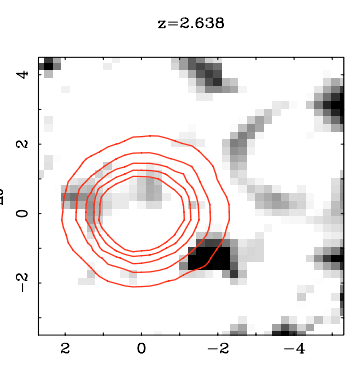

$\Delta \alpha$

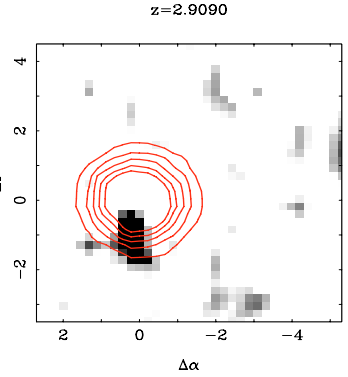

$\mathrm{z}=3.391$

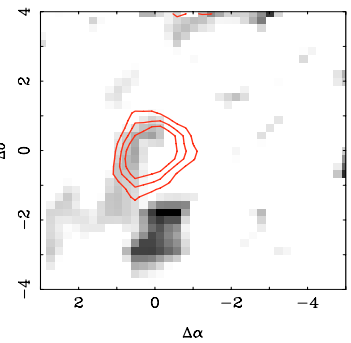

$\mathrm{z}=3.554$

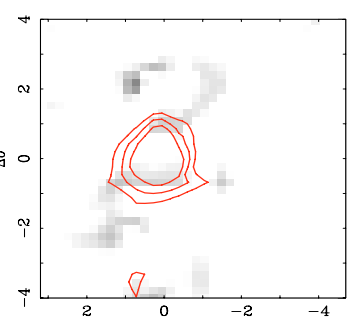

$\Delta a$

$z=3.762$

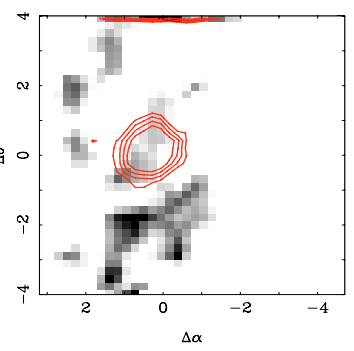

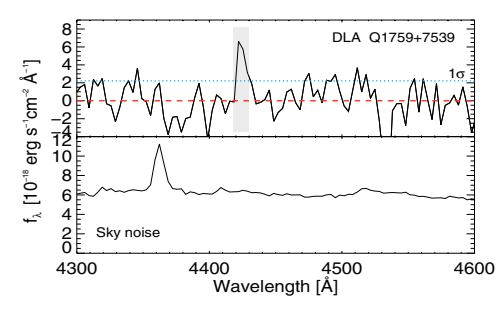
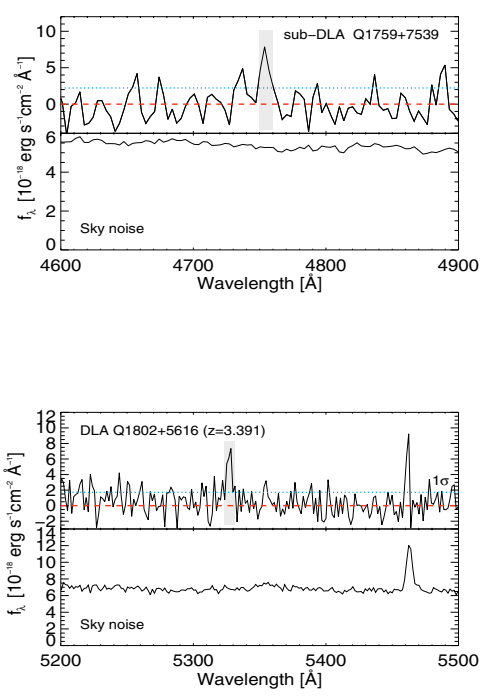

Fig. 2. continued.

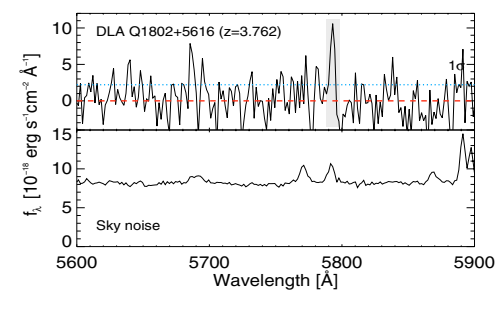


L. Christensen et al.: An IFS survey for high-z DLA galaxies, Online Material $p 4$

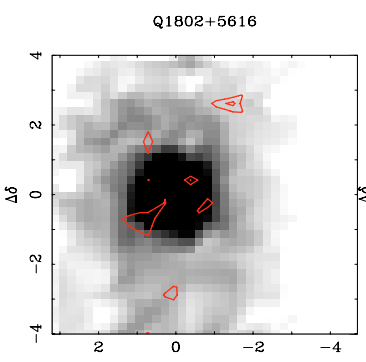

$\Delta \alpha$

Q2155+1358

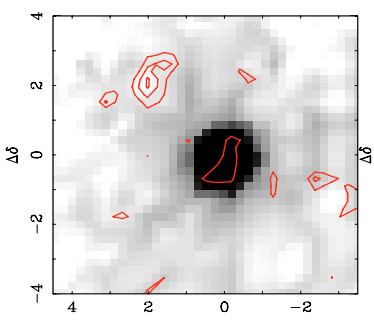

Q2155+1358

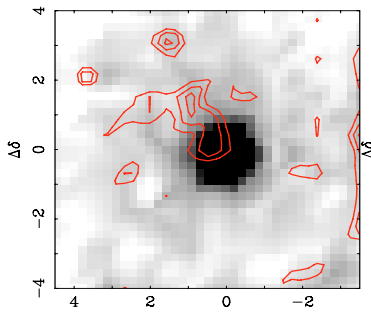

$\Delta a$

Q2155+1358

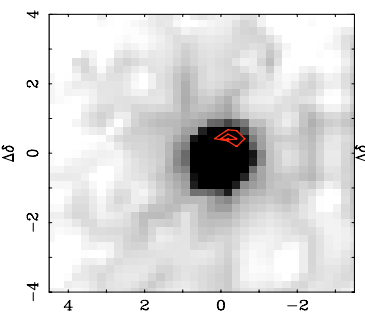

$\Delta \alpha$

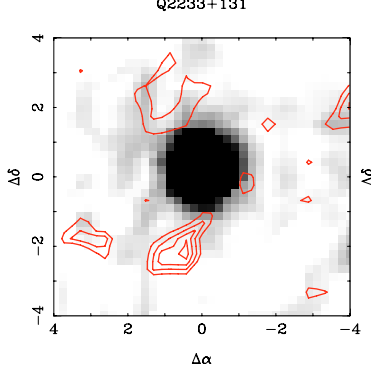

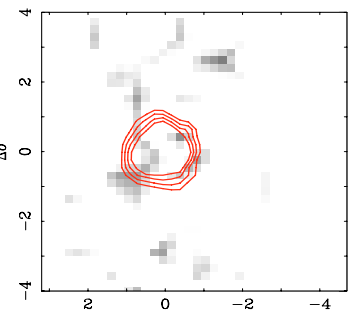

$\Delta a$

$z=3.142$

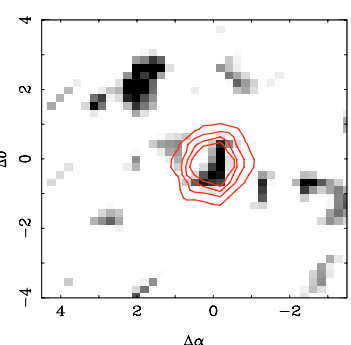

$z=3.317$

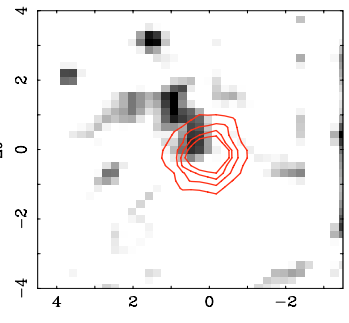

$\Delta a$
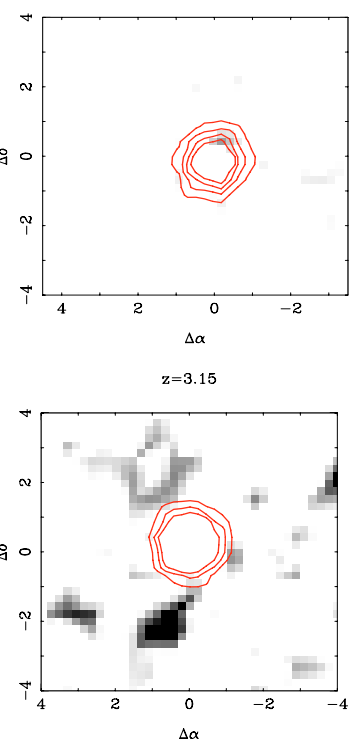
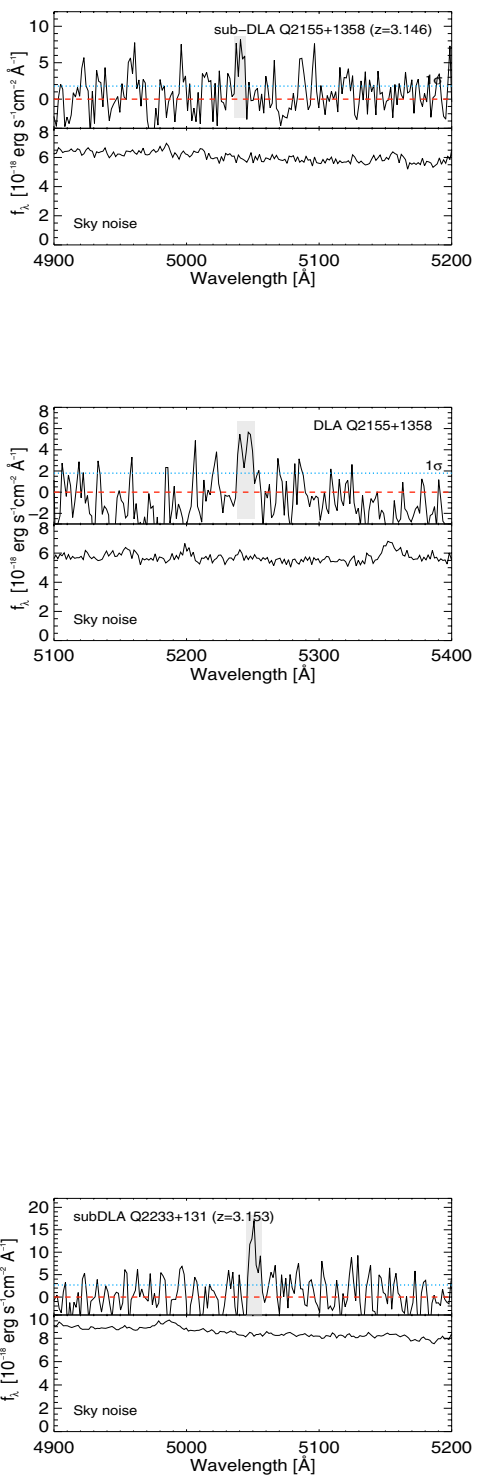

Fig. 2. continued. 
L. Christensen et al.: An IFS survey for high-z DLA galaxies, Online Material $p 5$

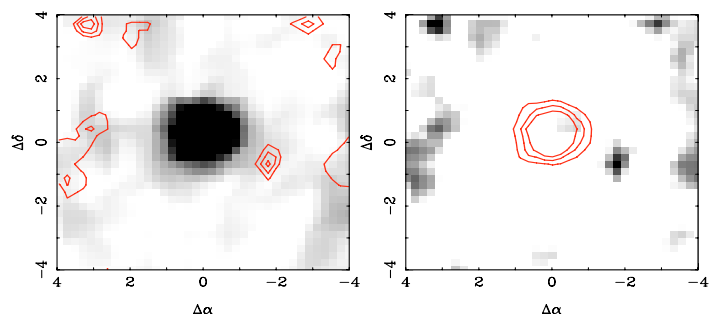

Fig. 2. continued. 